\title{
International Benchmarking of the Individual Tree Detection Methods for Modeling 3-D Canopy Structure for Silviculture and Forest Ecology Using Airborne Laser Scanning
}

\author{
Yunsheng Wang, Juha Hyyppä, Xinlian Liang, Harri Kaartinen, Xiaowei Yu, Eva Lindberg, \\ Johan Holmgren, Yuchu Qin, Clément Mallet, António Ferraz, Hossein Torabzadeh, \\ Felix Morsdorf, Lingli Zhu, Jingbin Liu, and Petteri Alho
}

\begin{abstract}
Canopy structure plays an essential role in biophysical activities in forest environments. However, quantitative descriptions of a 3-D canopy structure are extremely difficult because of the complexity and heterogeneity of forest systems. Airborne
\end{abstract}

Manuscript received August 13, 2015; revised January 9, 2016; accepted March 7, 2016. Date of publication June 16, 2016; date of current version August 2, 2016. This work was supported in part by the Finnish Academy through projects "Centre of Excellence in Laser Scanning Research (CoE-LaSR)" under Grant 272195, "Interaction of Lidar/Radar Beams with Forests Using Mini-UAV and Mobile Forest Tomography" under Grant 259348, and "Competence Based Growth Through Integrated Disruptive Technologies of 3-D Digitalization, Robotics, Geospatial Information and Image Processing/ Computing Point Cloud Ecosystem" under Grant 293389 and in part by the European Community's Seventh Framework Program (FP7/2007-2013) under Grant Agreement 606971. The work of Y. Qin, C. Mallet, and A. Ferraz was supported by the French National Research Agency through the FORESEE Project under Grant ANR-2010-BIOE-008. The work of A. Ferraz was supported by the Jet Propulsion Laboratory through the NASA Postdoctoral Program, which was administrated by the Oak Ridge Associated Universities through a contract with NASA. Y. Wang, J. Hyyppä, and X. Liang contributed equally to this work.

Y. Wang is with the Department of Remote Sensing and Photogrammetry, Finnish Geospatial Research Institute, FGI (former Finnish Geodetic Institute), 02431 Masala, Finland, with the Centre of Excellence in Laser Scanning Research, Academy of Finland, 00531 Helsinki, Finland, and also with the Department of Geography and Geology, Geography Section, University of Turku, 20014 Turku, Finland.

J. Hyyppä, X. Liang, H. Kaartinen, X. Yu, L. Zhu, and J. Liu are with the Department of Remote Sensing and Photogrammetry, Finnish Geospatial Research Institute, FGI (former Finnish Geodetic Institute), 02431 Masala, Finland, and also with the Centre of Excellence in Laser Scanning Research, Academy of Finland, 00531 Helsinki, Finland (e-mail: xinlian.liang@fgi.fi).

E. Lindberg and J. Holmgren are with the Department of Forest Resource Management, Swedish University of Agricultural Sciences, 90183 Umeå, Sweden.

Y. Qin and C. Mallet are with the MATIS, Université Paris-Est, IGN, 94160 Paris, France.

A. Ferraz is with the MATIS, Université Paris-Est, IGN, 94160 Paris, France, and also with the Jet Propulsion Laboratory, California Institute of Technology, Pasadena, CA 91109 USA.

$\mathrm{H}$. Torabzadeh is with the Remote Sensing Laboratories, University of Zurich, CH-8057 Zurich, Switzerland, and also with the Department of Civil Engineering, Bu-Ali Sina University, Hamedan 65178-4161, Iran.

F. Morsdorf is with the Remote Sensing Laboratories, University of Zurich, CH-8057 Zurich, Switzerland.

P. Alho is with the Department of Remote Sensing and Photogrammetry, Finnish Geospatial Research Institute, FGI (former Finnish Geodetic Institute), 02431 Masala, Finland, and also with the Department of Geography and Geology, Geography Section, University of Turku, 20014 Turku, Finland.

Color versions of one or more of the figures in this paper are available online at http://ieeexplore.ieee.org.

Digital Object Identifier 10.1109/TGRS.2016.2543225 laser scanning (ALS) provides an opportunity to automatically measure a 3-D canopy structure in large areas. Compared with other point cloud technologies such as the image-based Structure from Motion, the power of ALS lies in its ability to penetrate canopies and depict subordinate trees. However, such capabilities have been poorly explored so far. In this paper, the potential of ALS-based approaches in depicting a 3-D canopy structure is explored in detail through an international benchmarking of five recently developed ALS-based individual tree detection (ITD) methods. For the first time, the results of the ITD methods are evaluated for each of four crown classes, i.e., dominant, codominant, intermediate, and suppressed trees, which provides insight toward understanding the current status of depicting a 3-D canopy structure using ITD methods, particularly with respect to their performances, potential, and challenges. This benchmarking study revealed that the canopy structure plays a considerable role in the detection accuracy of ITD methods, and its influence is even greater than that of the tree species as well as the species composition in a stand. The study also reveals the importance of utilizing the point cloud data for the detection of intermediate and suppressed trees. Different from what has been reported in previous studies, point density was found to be a highly influential factor in the performance of the methods that use point cloud data. Greater efforts should be invested in the point-based or hybrid ITD approaches to model the 3-D canopy structure and to further explore the potential of high-density and multiwavelengths ALS data.

Index Terms-Airborne laser scanning (ALS), benchmark, canopy structure, crown class, individual tree detection (ITD), LiDAR, point cloud, subordinate tree.

\section{INTRODUCTION}

C ANOPY structure plays an essential role in biophysical activities in forest environments. The presence and structure of canopies exert a major influence on the temperature, vapor concentration, and radiation regime in forests. The interception and transmission of precipitation are also affected, as are soil temperature and soil heat flow [1]. At an individual tree level, canopy structure refers to tree position, size, and 3-D geometry. At a stand/forest level, canopy structure is depicted by the relative distribution of the tree crowns with respect to their neighboring trees, i.e., the distribution of crown classes of individual trees in a stand/forest. The most classic classification of crown class consists of four distinct classes [2], namely, dominant, codominant, intermediate, 
and suppressed canopy, which refer to the exposure of tree crowns to direct sunlight.

The quantitative description of 3-D canopy structure is, however, extremely difficult because of the complexity and heterogeneity of forest systems. Measurements of 3-D crown structure using conventional methods in the field are laborious and often imprecise [3]. Two-dimensional features, e.g., leaf area index (LAI) and canopy closure, are practically used as surrogates for 3-D canopy geometries. Even with the use of 2-D measurements, it remains challenging to reliably assess forest canopy structures across a wide range of conditions. For example, direct LAI measurements in the field are labor and cost intensive, and even nondestructive methods can disturb the canopy to various extents. Indirect measurements are conventionally collected using 2-D photographs. The interpretation of hemispherical fish-eye photographs is based on spectral reflections, which are influenced by sky conditions and many other factors related to image capturing. Therefore, observations are less consistent between different studies. In addition, characterizing canopy structure in large areas using ground-based tools is practically impossible because it requires intensive field surveys, and the high cost of such campaigns is not affordable for most applications.

It will be a significant advance if the 3-D canopy structure over large areas can be automatically and reliably measured from airborne platforms. Airborne laser scanning (ALS) is a promising active remote sensing technique to provide direct, accurate, and detailed 3-D measurements of forest areas. The technology has been well accepted in forest-related studies and applications during the last two decades, e.g., [4]-[6]. Two groups of ALS-based approaches are used to assess forest attributes, i.e., area-based approaches (ABAs) and individual/ single tree detection (ITD) approaches. ABAs rely on the correlation between field-measured attributes and ALS-derived predictors to produce statistical predictions of forest variables [6], [7]. For the vertical canopy structure, ABAs are capable of detecting and mapping the distribution of the secondary canopy layer by statistical analysis of the height distribution of the ALS points [8]. When using ITD approaches, individual trees are detected and modeled using ALS data to extract treelevel variables [9]-[11]. ITD approaches are superior for studies on canopy structure because they provide information at an individual tree crown level.

ALS-based ITD approaches can be divided into three typical types, namely, raster-based, point-based, and hybrid approaches. Raster-based ITD approaches work on rasterized data that are interpolated from the original ALS point cloud. Each pixel in the rasterized data corresponds to certain averaged attributes or classified information of the point cloud, such as the maximum, minimum, or mean height of the ALS points, or different returns (echoes) of the laser pulses [12]. The most commonly used raster data is the crown height model (CHM), which is generated based on the normalized height (above terrain height) of the ALS points. The point-based ITD approach directly models 3-D individual crown shape from ALS point cloud [8]. This approach is capable of modeling trees in both the topmost and secondary canopy layers because it uses the complete ALS point cloud. Some recent efforts attempted to com- bine raster-based and point-based ITD approaches, i.e., hybrid ITD approaches [13], [14], where individual trees or groups of trees are detected in the rasterized data, and the 3-D canopy of the detected crowns is then modeled from the segmented points.

Although ALS has been practically used in forest inventories for more than a decade [15], the potential of this technology has not yet been fully explored. Compared with other point cloud technologies such as the image-based Structure from Motion, the power of ALS lies in its ability to penetrate canopies, to characterize the 3-D canopy structure, and to support further 3 -D interpretation of forest ecosystems. However, such capabilities have been poorly explored so far. To reduce the computational complexity of data processing, ITD studies have focused primarily on raster-based methods thus far [16]. However, vertical canopy information is entirely or mostly lost in the rasterized data. It is therefore difficult to use raster-based approaches to characterize vertical canopy structures and subordinate trees.

In [17] and [18], 11 and 13 methods (e.g., from Finland, Germany, Norway, Sweden, Switzerland, and the United States) were compared for their performance in a boreal forest using ALS data with different point densities. The comparison revealed that the point-based methods yielded better results with respect to detecting subordinate trees in comparison with rasterbased methods. However, the methodological developments for detecting subordinate trees (i.e., intermediate and suppressed trees) are clearly inadequate. In [19], six methods from Finland, Germany, Norway, and Sweden were compared across five forest sites. The research showed that the forest structure was the main factor in determining the performance of the methods. In [20], eight methods from Austria, France, Italy, and Slovenia were compared in Alpine forests. The study confirmed that the vertical structure of the forest canopy significantly impacts the detection accuracy of ITD approaches.

Results from all these previous comparisons indicate that the smaller and subordinate trees are among the main challenges of applying the ITD approach to measure forest structure. However, detailed analyses on the measurement of trees in the secondary canopy layer have not yet been made. The performance of ALS and the ITD approaches in characterizing 3-D canopy structures, i.e., the detection accuracy of trees in different crown classes, remains unclear. Meanwhile, the need to acquire detailed 3-D canopy structure information is rapidly increasing. It is therefore necessary to further explore the potential of ALS and to advance the ALS-based ITD approaches beyond the topmost canopy layer. Future studies require a clear understanding of the current status of the ITD methods, particularly with respect to their ability to detect subordinate trees. The challenges of applying ITD approaches in modeling 3-D canopy structure should also be clarified.

This paper explores the potential of measuring 3-D canopy structure from ALS data through an international benchmark of five recently developed ITD methods. For the first time, the detection accuracy is separately evaluated for different crown classes within stands, such that deeper insight toward understanding the capability of recent ALS-based ITD methods for depicting 3-D canopy structure can be achieved. Section II describes the test sites, as well as the ALS and reference data. Section III gives the detailed information of the five compared 
TABLE I

Detailed Stand Information of the Five Test Plots

\begin{tabular}{|c|c|c|c|c|c|c|c|c|c|c|c|c|c|}
\hline \multirow[t]{3}{*}{ Plot } & \multirow[t]{3}{*}{$\begin{array}{l}\text { Size } \\
\text { (ha) }\end{array}$} & \multirow[t]{3}{*}{$\begin{array}{c}\text { Forest } \\
\text { type }\end{array}$} & \multicolumn{6}{|c|}{$\begin{array}{c}\text { Species composition } \\
(\%)\end{array}$} & \multirow[t]{3}{*}{$\begin{array}{c}\text { Density } \\
\text { (stems/ha) }\end{array}$} & \multicolumn{4}{|c|}{$\begin{array}{c}\text { Tree Height } \\
\text { (m) }\end{array}$} \\
\hline & & & Conife & erous & & eciduou & & Bush & & Min. & Max. & Mean. & Median \\
\hline & & & Spruce & Pine & Aspen & Birch & Other & & & & & & \\
\hline A1 & 0.12 & Deciduous & 15.0 & 10.0 & 27.5 & 30.0 & 15.0 & 2.5 & 333 & 3.7 & 26.2 & 13.3 & 14.0 \\
\hline A2 & 0.24 & Mixed & 45.5 & 8.9 & 12.9 & 19.8 & 10.9 & 2.0 & 421 & 1.7 & 26.0 & 13.8 & 13.4 \\
\hline B1 & 0.16 & Coniferous & 80.0 & 7.2 & 1.8 & 5.4 & 5.6 & - & 350 & 3.0 & 24.2 & 16.8 & 19.5 \\
\hline B2 & 0.32 & Coniferous & 55.4 & 17.9 & 0.8 & 13.4 & 8.1 & 4.4 & 353 & 1.8 & 27.2 & 16.1 & 19.1 \\
\hline B3 & 0.04 & Coniferous & 2.5 & 83.3 & - & 7.1 & 7.1 & - & 1050 & 1.3 & 12.6 & 5.0 & 4.2 \\
\hline
\end{tabular}

ITD approaches. Among them, three (i.e., two raster-based approaches from France and Sweden and one hybrid approach from Sweden) were published in previous scientific literatures, one method (i.e., a hybrid approach from Switzerland) was upgraded after the first publication and the new advances have not been published, and one method (i.e., a point-based approach from Finland) was newly developed and have not yet been published in any other literature. Section IV evaluates the performances of the compared methods with respect to four crown classes. Section V further analyzes the performance of the different methodologies (i.e., raster-based, hybrid, and pointbased) and the impacts of different forest stand factors (i.e., the species composition, stand age/developmental stage, and stem density). The section also discusses the capability of the applied first-last discrete return ALS to detect suppressed trees in the secondary canopy layer and the challenges associated with using the ITD methods to detect subordinate trees. Section VI summarizes all the important findings of this benchmarking.

\section{Materials}

The ALS and reference data used in this study are the same data set as that used in [17] and [18] to maintain the consistency of this benchmarking with the previous benchmarking of the "Tree Extraction" project organized by the European Spatial Data Research Organization and the International Society for Photogrammetry and Remote Sensing. Other than the compared methods, the main differences between this benchmarking and the other previous comparisons are, first, that for the first time, the detection accuracy is separately evaluated with respect to different crown classes and, second, that the impact of different forest stand situations is thoroughly investigated. Thus, a deeper insight into recent ALS-based ITD methods for depicting 3-D canopy structure can be achieved.

\section{A. Forest Sites and Test Plots}

The study area is located in southern Finland, approximately $18 \mathrm{~km}$ west of Helsinki, with partly flat and partly steep (max. elevation difference of $45 \mathrm{~m}$ ) terrain. The main tree species in the forest area are Scots pine (Pinus sylvestris L.), Norway spruce (Picea abies L.), Silver and Downy birches (Betula sp. L.), and Aspen (Populus tremula L.). The five test plots have diverse forest stands: Trees are at various growth stages, and the species composition varies from a relatively homogenous coniferous or deciduous to a mixed forest. Detailed information on plot size, stem density, species composition, and tree height of each test plot is presented in Table I. The locations of the five test plots are shown in Fig. 1.

Plots A1, A2, B1, and B2 have a similar tree height range, but plots $\mathrm{A} 1$ and $\mathrm{A} 2$ have clearly smaller mean tree heights than plots $\mathrm{B} 1$ and $\mathrm{B} 2$, which indicates that more small trees exist in plots A1 and A2. Meanwhile, plots A1 and A2 have an almost evenly distributed population at different tree heights, which indicates that the vertical structure is rich but the canopy layers can hardly be recognized using statistical analysis, i.e., tree height histograms. Plot B1 is an old coniferous stand with tall trees of similar tree heights. Plot B2 is a mature but still developing stand that consists of trees at different growth stages. Plot B3 is a very young and dense stand.

\section{B. ALS and Reference Data Acquisition}

ALS data were collected in June 2004 using an Optech ALTM 2033 system (Optech Incorporated, Toronto, ON, Canada) flown $600 \mathrm{~m}$ above ground level and operating at a pulse frequency of $33 \mathrm{kHz}$. Four flight strips were conducted, and three point clouds of different point densities $\left(2,4\right.$, and $\left.8 \mathrm{pts} / \mathrm{m}^{2}\right)$ were produced from different combinations of the flight strips.

Field reference data were collected from TLS data. Five field plots were scanned at 48 locations using a Faro LS880HE scanner (FARO USA, Lake Mary, FL, USA). The locations, species, and heights of 352 trees were manually measured from the TLS data. The location of the test sites, test plots, and TLS reference data collection points are shown in Fig. 1.

\section{Crown Classes of Reference Trees}

To investigate the capability of the compared ITD methods for depicting the 3-D canopy structure, the reference trees in the five test plots were classified into four crown classes based on their positions relative to the adjacent tree crowns. According to the classic definition of crown classes (Kraft, 1884), the "Dominant" trees are those with the best access to sunlight. The "Codominant" trees have their treetops exposed to direct sunlight. The "Intermediate" trees have limited access to direct sunlight, i.e., only parts of their crowns. The "Suppressed" trees are overtopped and have minor access to sunlight. In practice, 

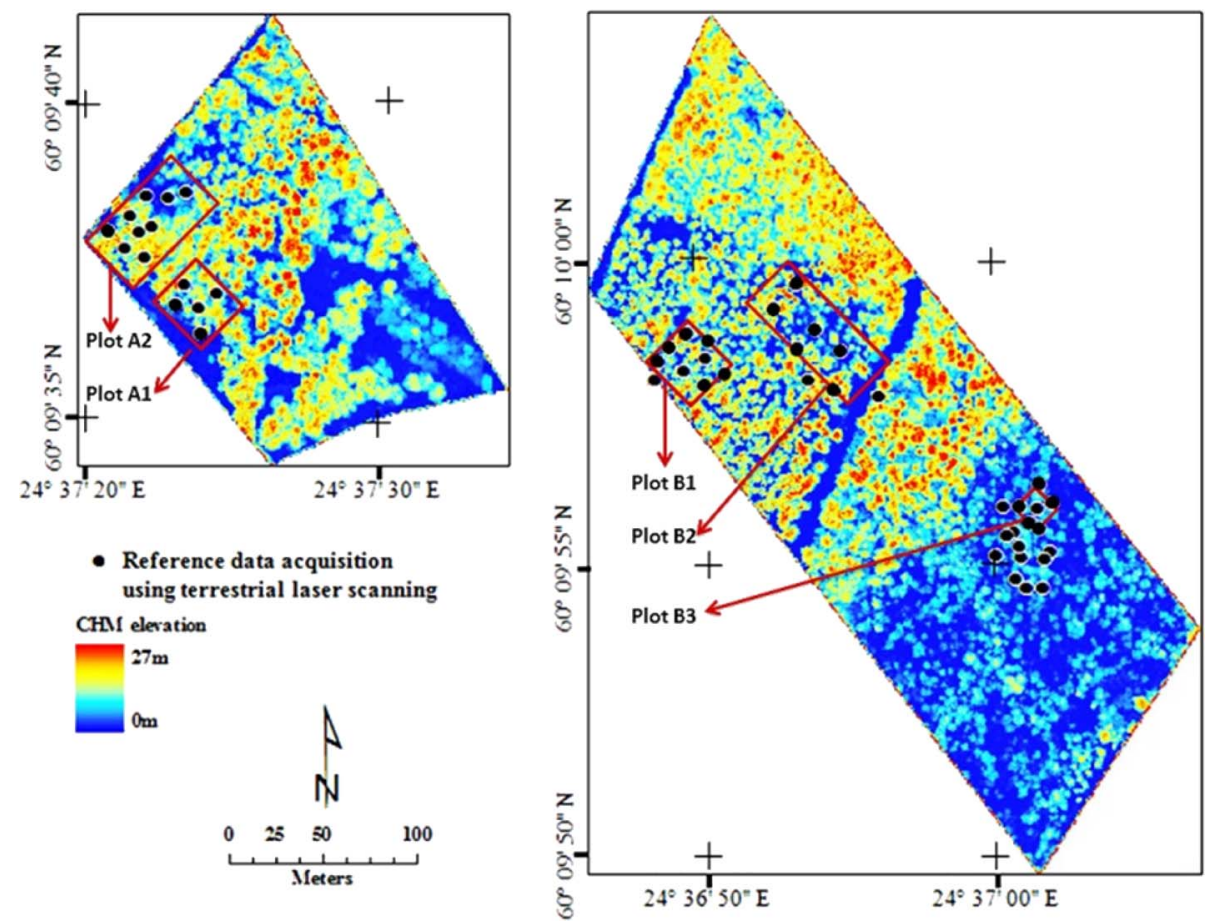

Fig. 1. Five test plots from two test sites with reference information derived from TLS data. ALS data of two test sites A (left) and B (right) are colored according to the canopy height. Black dots represent the locations of the TLS measurements.

TABLE II

Population (\%) of Different Crown Classes in Each Test Plot

\begin{tabular}{cccccc}
\hline Crown Class & A1 & A2 & B1 & B2 & B3 \\
\hline Dominant & 37.5 & 21.8 & 57.1 & 48.7 & 33.3 \\
Co-dominant & 10.0 & 29.7 & 16.1 & 26.5 & 26.2 \\
Intermediate & 22.5 & 25.7 & 21.4 & 12.4 & 14.3 \\
Suppressed & 30.0 & 22.8 & 5.4 & 12.4 & 26.2 \\
$\quad$ Sum & 100 & 100 & 100 & 100 & 100 \\
\hline
\end{tabular}

these qualitative definitions have been quantitatively expressed [18] as follows:

Dominant (Dom): tallest trees in the neighborhood or isolated trees that have a 2-D distance to the closest neighboring tree that exceeds $3 \mathrm{~m}$;

Codominant $(\mathrm{CoD})$ : trees in a group of similar trees, where the 2-D distance between these trees and the closest neighbor is less than $3 \mathrm{~m}$;

Intermediate (Int): trees located next to a larger tree and whose crowns are partly covered;

Suppressed (Sup): trees located under a larger tree and whose crowns are totally covered by neighboring crowns.

The distribution of different crown classes in each test plot is shown in Table II.

The distribution of crown classes describes the canopy structure and indicates the development and regeneration situation of a forest. Plots B1 and B2 are mature stands with the highest population of dominant trees and with less regeneration. Plot B1 has the smallest population of suppressed trees. Plot B2 has the smallest population of intermediate trees. The other three test plots are developing stands that have an approximately even distribution of crown classes.

Tree crown class is a relative and local concept that describes the crown structure at a specific location. For example, a dominant tree is the tallest tree in its neighborhood, but it may not be the tallest in the whole stand. The tree height distribution of the crown classes in each test plot is presented in Table III. The minimum heights of the dominant trees in plots A2, B2, and $\mathrm{B} 3$ are all below $5 \mathrm{~m}$, and the maximum heights are approximately $20 \mathrm{~m}$, which suggests high variance in the overall canopy height in these three plots. Plots A1 and A2 have a high population of suppressed trees with a relatively high mean height $(8.5$ and $7.0 \mathrm{~m})$, indicating strong regeneration and a multilayered structure.

The composition of tree species in different crown classes provides greater insight into the stand structures. A detailed species-specific crown class composition of each test plot is given in Table IV. In plot A1, the coniferous population is significant with respect to its codominant trees although the stand itself is deciduous dominated. Most of the subordinate trees in $\mathrm{A} 1$ are deciduous. Plot A2 is a mixed stand with a large deciduous population in its dominant trees and a balanced distribution of coniferous and deciduous species in its codominant trees. The regeneration in plot A2 is mainly composed of coniferous species. Plots B1 and B3 are almost pure-coniferous stands with a dominant coniferous population in all crown classes. In plot B2, approximately one-fourth of the species in each crown class are deciduous.

In the comparisons and analyses, the results of the five methods were evaluated according to the four crown classes. The influence of crown class and species on the performance of the ITD methods is discussed in detail in Section V-A. 
TABLE III

Tree Height (M) Distribution of Different Crown Classes in Each Test Plot

\begin{tabular}{|c|c|c|c|c|c|c|c|c|c|c|c|c|c|c|}
\hline $\begin{array}{l}\text { Crown } \\
\text { Class }\end{array}$ & Min. & $\begin{array}{c}\text { A1 } \\
\text { Max. }\end{array}$ & Mean & Min. & $\begin{array}{l}\text { A2 } \\
\text { Max. Mean }\end{array}$ & Min. & $\begin{array}{c}\text { B1 } \\
\text { Max. }\end{array}$ & Mean & Min. & $\begin{array}{c}\text { B2 } \\
\text { Max. }\end{array}$ & Mean & Min. & $\begin{array}{c}\text { B3 } \\
\text { Max. }\end{array}$ & Mean \\
\hline Dominant & 10.3 & 26.2 & 19.3 & 4.2 & 26.021 .4 & 3.4 & 24.2 & 20.1 & 3.1 & 27.2 & 19.1 & 1.3 & 9.9 & 5.3 \\
\hline Co-dominant & 15.1 & 18.2 & 16.4 & 8.0 & $23.8 \quad 16.3$ & 15.4 & 23.0 & 20.1 & 1.8 & 25.1 & 18.5 & 2.3 & 12.6 & 8.2 \\
\hline Intermediate & 3.7 & 17.7 & 8.2 & 1.7 & 17.610 .6 & 3.0 & 13.4 & 8.3 & 4.3 & 17.5 & 9.5 & 2.1 & 5.0 & 3.4 \\
\hline Suppressed & 4.3 & 14.6 & 8.5 & 2.4 & $15.0 \quad 7.0$ & 3.5 & 7.2 & 5.6 & 2.9 & 9.6 & 5.5 & 1.5 & 4.7 & 2.2 \\
\hline
\end{tabular}

TABLE IV

Composition (\%) of Tree SPECIES IN DifFERENT CRown Classes

\begin{tabular}{ccccccccccc}
\hline $\begin{array}{c}\text { Crown } \\
\text { Class }\end{array}$ & \multicolumn{2}{c}{ A1 } & \multicolumn{2}{c}{ A2 } & \multicolumn{2}{c}{ B1 } & \multicolumn{2}{c}{ B2 } & \multicolumn{2}{c}{ B3 } \\
\hline Dominant & 13.3 & 86.7 & 18.1 & 81.9 & 84.3 & 15.7 & 74.5 & 25.5 & 92.9 & 7.1 \\
Co-dominant & 75 & 25 & 53.3 & 46.7 & 100 & 0 & 73.3 & 26.7 & 63.6 & 36.4 \\
Intermediate & 22.2 & 77.8 & 73.1 & 26.9 & 91.6 & 8.4 & 78.6 & 21.4 & 100 & 0 \\
Suppressed & 25 & 75 & 69.6 & 30.4 & 66.7 & 33.3 & 64.3 & 35.7 & 90.9 & 9.1 \\
\hline${ }^{1}$ Coniferous; ${ }^{2}$ Deciduous & & & & & & & & & &
\end{tabular}

TABLE V

METHODS FOR COMPARISON

\begin{tabular}{llll}
\hline Method & Type & Partner & Country \\
\hline IGN-R & Raster-based & Laboratoire MATIS, Université Paris Est (IGN) & France \\
SLU-R & Raster-based & Swedish University of Agricultural Sciences (SLU) & Sweden \\
SLU-H & Hybrid & Swedish University of Agricultural Sciences (SLU) & Sweden \\
UZH-H* & Hybrid & University of Zürich (UZH) & Switzerland \\
FGI-P** & Point-based & Finnish Geospatial Information Institute (FGI) & Finland \\
\hline${ }^{*}$ Unpublished improvements & & \\
${ }^{* *}$ New, unpublished method & &
\end{tabular}

\section{METHODS}

The same ALS data set was processed using five methods, and their results were compared to evaluate their performances. The five compared methods are listed in Table V. All these five methods are newly developed after the previous comparisons conducted in [17]-[19], and the authors of the methods are willing to participate in this benchmarking. Methods are named after the institute abbreviation of their developers. The last letter in a method's name refers to the type of driven data applied in the method, i.e., raster-based (R), point-based (P), and hybrid $(\mathrm{H})$.

The IGN-R, SLU-R, and SLU-H methods were previously published in the scientific literature. The SLU-H method was also evaluated in [20], where it was referred to as "SLU." UZH-H is an updated version of the "Zürich" method that was evaluated in the previous comparison conducted by Kaartinen et al. in [18]. Several improvements have been made for this method after the previous comparisons; however, not all of the new advances have been published. The FGI-P method is a newly developed point-based algorithm that has not yet been published.

\section{A. Methods From Collaborators}

Brief descriptions of the methods used by collaborators are given in Section III-A. Detailed information on the FGI-P method is presented in Section III-B.
1) IGN-R: The IGN-R method [21] is a raster-based ITD algorithm. Based on the assumption that a tree only has one treetop, this multiscale gradient transformation approach was proposed to highlight the elevation characteristics of a CHM generated from an ALS point cloud over the tree canopies. The method consists of three main steps: 1) the calculation of the gradient comparison ratio (GCR) and the accumulation of GCRs of different window sizes; 2) local maxima (LMs) detection of the GCR accumulation using a flood-fill algorithm to provide initial markers for tree segmentation; and 3) markercontrolled watershed segmentation for ITD. For a given pixel, the GCR value for a particular window size was defined as the ratio between the number of pixels with a lower elevation in the window and the number of overall pixels in the window. The GCR was proposed to provide a normalized indicator to describe the elevation characteristics of individual tree crowns without the consideration of absolute tree height. The IGN-R method was developed to provide initial parameters for a pointbased tree detection algorithm using mean-shift clustering [13]. The window sizes for GCR calculation were $3,5,7, \ldots, 17$, with a pixel size of $0.5 \mathrm{~m}$. Using the parameters provided for this experiment, the goal of the IGN-R method was not to achieve high detection rates but to accurately extract dominant tree crown samples at the forest stand level.

2) SLU-R: In the SLU-R method [22], the automatic ITD procedure consists of a training phase and a prediction phase. In this paper, the training phase was different compared with that in [22], and another probability function was used. For the 
training phase, the segmentation algorithm was first applied to sample plots that contained manually digitized tree positions. The result from the first segmentation, i.e., number of trees within each segment, was used to calculate the probability that a segment contained only one tree as a function of the variables derived from the ALS data, i.e., the ratio between crown radius and tree height. The probability function that was estimated in the first phase was used in a merging step of the algorithm in the second phase. The segmentation algorithm consisted of four steps. 1) The first step is creating a CHM from the ALS data. 2) The second step is calculating a correlation surface, i.e., a raster, based on the CHM. For each pixel in the correlation surface, a correlation coefficient was calculated between a best fit ellipsoid that was constrained by the CHM height at the corresponding pixel and the ALS points that were located in the same neighborhood defined by the best fit ellipsoid. The higher the coefficient of a pixel, the higher the likelihood that the pixel represents a treetop. 3) The third step is segmentation of the correlation surface. An individual tree crown was segmented from the correlation surface using the watershed algorithm. 4) The fourth and final step is merging segments based on the probability function that was generated in the training phase. Following the segmentation approach, it was expected that each segment represented one individual tree crown. All raster had a 0.25-m pixel size for this experiment.

3) SLU-H: The SLU-H method [14] is an extended approach based on the outcomes of the SLU-R method. The results of the SLU-R method, i.e., the segments of the correlation surface model, were used as an initial input for the 3-D delineation of individual tree crowns in the ALS point data. Threedimensional tree crowns were detected and modeled from the point cloud using prior knowledge about the shapes and proportions of the tree crowns, i.e., so-called ellipsoidal tree model clustering. The method used more information derived from the segmentation of the correlation surface model by initially defining the clusters in the topmost canopy layer as the ALS points assigned to each segment.

The delineation of individual trees was achieved in two steps. First, tree crowns in the topmost canopy layer were delineated by segmentation of a correlation surface model using the SLU-R method; in the second step, ellipsoidal tree model clustering of the ALS points was conducted in 3-D to extract the point cluster of each detected individual tree crown. The clustering was aimed at extracting tree crown clusters from both tree crowns in the topmost canopy layer as well as smaller trees and larger shrubs below. The algorithm was based on K-means clustering using ellipsoidal tree crown models. The clustering approach not only enabled ITD in understory layers but also improved the ITD results in the topmost canopy layer based on the SLU-R method.

4) $U Z H-H$ : This method is an extended version of a method that was published in [23], which was referred to as the "Zürich" method in [18]. In this extended version, the ALS point cloud was gradually divided into potential trees by sequentially applying three different segmentation approaches.

First, the inverted CHM was segmented by applying a watershed transformation. In addition to four parameters to control the CHM quality (i.e., resolution, search radius, and the size and scale of a lowpass filter), two more parameters were introduced: the minimum watershed size for removing tiny watersheds and the minimum height of trees to exclude bushes. Second, the corresponding ALS points within each CHM segment were separated into different levels by identifying large gaps in the vertical distribution of the ALS points, which resulted in the differentiation between the topmost and secondary canopy layers. This procedure was controlled by adjusting the minimum number of points per vertical layer and the minimum height for defining a vertical gap. In a third step, the ALS points were clustered to individual tree crowns. The clustering approach was based on Gaussian mixture models such that all the candidate points were separated based on the mean values and covariance matrices describing the spatial distribution of the ALS points for all three coordinate axes. The LM points of the CHM were transformed into a cylindrical coordinate space, and only LMs with sufficient ALS points in their vicinity were retained as seed points for the clustering approach. Following a kernel density analysis, clusters were identified by analyzing the local point cloud density. Through iterative application of the sequential steps, clusters of individual tree crowns from both the topmost and secondary canopy layers can be detected. The CHM was consecutively updated during the iterations. The pixel size of the CHM was $1 \mathrm{~m}$ for this experiment, and a $5 \times 5$ Gaussian filter was applied for CHM smoothing.

The clusters were considered to represent individual tree crowns if certain criteria, e.g., minimum crown area, were satisfied. The center of gravity of the points belonging to each cluster specifies the tree position $(x, y)$. The maximum and minimum heights of the points of a cluster were considered as the tree height and the crown base height, respectively.

\section{B. New Point-Based Method (FGI-P)}

The individual tree extraction method developed by the Finnish Geospatial Research Institute (FGI) is a new pointbased approach. The original ALS point cloud is first normalized using a Digital Terrain Model (DTM) and is then transferred to a local voxel space. For each voxel that contains at least one ALS point, a vox-point is generated using the center location of the corresponding voxel as its coordinates. Thus, a new vox-point cloud is created in the voxel space, which is referred to as a "vox-cloud" in the following text. Treetops are detected in the vox-cloud by studying the 3-D spatial distribution of the vox-points. Treetops from different canopy layers are simultaneously detected. Tree crowns of individual trees are extracted from the vox-cloud in a bottom-up order. Attributes such as the location of the treetop, the tree height, the crown width, and the crown depth are derived after the tree crown delineation.

1) Generation of a Vox-Cloud From an ALS Point Cloud: The original ALS point cloud is normalized using a DTM and is transferred to a local voxel space using a given resolution. In this paper, the voxel resolution is $0.5 \mathrm{~m} \times 0.5 \mathrm{~m} \times 0.5 \mathrm{~m}$, which was a quite robust voxel size for the point densities of 2,4 , and $8 \mathrm{pts} / \mathrm{m}^{2}$. A vox-point in the vox-cloud is the center position of the voxel element that has at least one ALS point. The number of ALS points that are located inside the voxel element is recorded as the weight of the vox-point. The vox-cloud presents 


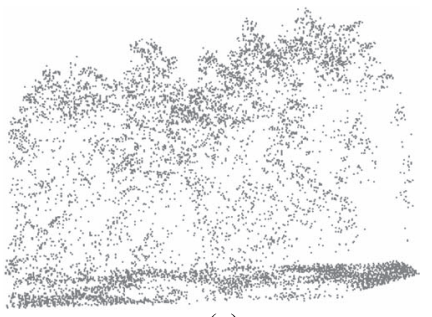

(a)

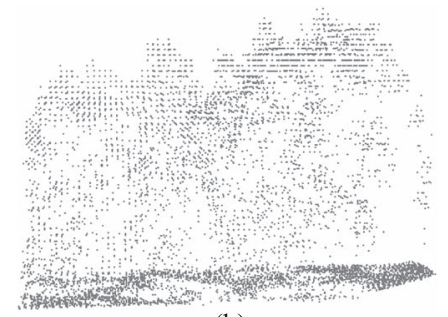

(b)

Fig. 2. Example of the (a) original ALS point cloud and (b) the corresponding vox-cloud.

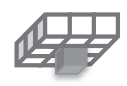

SE1 $(3 \times 3 \times 1$ above $)$

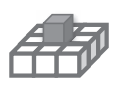

SE5 $(3 \times 3 \times 1$ below $)$

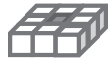

SE9 (8 surrounding) SE10 (24 surrounding)

Fig. 3. Ten 3-D SEs defined as treetop detectors. The solid gray cube represents the voxel element whose center point is the vox-point to be inspected.

a forest structure that is highly similar to the original ALS point cloud, as shown in Fig. 2. The major advantage of the vox-cloud compared with the original point data is the easy access to the neighborhood of each vox-point. For example, neighboring vox-points of a vox-point $V(x, y, z)$ are simply $V_{n}(x+i, y+j, z+k)$, where $i, j$, and $k$ indicate the directions and distances of the neighboring vox-points of the voxpoint $V(x, y, z)$. Therefore, the study of the local distribution of a point cloud at a certain location is much more convenient using the vox-cloud.

2) Detection of Treetops: Treetops are supposed to have three main morphological characteristics in 3-D space according to the basic principles of tree growth. First, there is certain open space above the tree crowns, and treetops are the highest points in the neighborhood, i.e., LMs. The difference between the dominant trees and the subordinate trees is that they have different sizes of open space above their crowns. Second, treetops are supported by the crowns. There is a cluster of ALS points describing a tree crown in the neighborhood of a treetop. Third, a treetop cannot be spatially attached to another treetop, or there must be a certain distance between individual treetops in 3-D. Based on these three morphological characteristics, treetops can be detected in all canopy layers in two steps by studying the distribution of vox-points.

In the first step, candidate treetops are detected according to the aforementioned first and second morphological characteristics. As shown in Fig. 3, ten basic structure elements (SEs) are defined as treetop detectors, which represent a different location, shape, and size of the neighborhood surrounding a vox-point to be inspected.

The shape and size of an inspected neighborhood can be defined by one SE or the combination of several SEs. For each vox-point, the distribution of the surrounding vox-points
TABLE VI

Constraints on CAndidate Treetop VoX-Point SElection

\begin{tabular}{|c|c|}
\hline Height level & Constrains for candidate treetop vox-points \\
\hline$>20 \mathrm{~m}$ & $\mathrm{SE} 4=0 \& \mathrm{SE} 5>=2 \& \mathrm{SE} 8>=5$ \\
\hline 15-20 m & $\mathrm{SE} 3+(\mathrm{SE} 2-\mathrm{SE} 1)=0 \& \mathrm{SE} 5+(\mathrm{SE} 8-\mathrm{SE} 7)>=4 \& \mathrm{SE} 10<=1$ \\
\hline $10-15 \mathrm{~m}$ & $\mathrm{SE} 2=0 \& \mathrm{SE} 5+(\mathrm{SE} 8-\mathrm{SE} 7)>=2 \& \mathrm{SE} 10<=2$ \\
\hline 5-10 m & $\mathrm{SE} 1=0 \&$ SE $8>=3 \&$ SE $9<=1$ \\
\hline $1-5 \mathrm{~m}$ & $\mathrm{SE} 1=0 \& \mathrm{SE} 8>=2$ \\
\hline
\end{tabular}

in a neighborhood is estimated by the number of surrounding vox-points in the space defined by the SEs. For an inspected vox-point, the number of surrounding vox-points in the space defined by an SE is assigned as the weight of the SE. For example, if two vox-points exist in the nine connecting spaces defined by SE1 of a vox-point, a weight value of 2 is assigned to SE1 of the vox-point.

An attribute table is generated for the vox-cloud, in which each vox-point has ten weight values for the ten SEs that represent the distribution of the surrounding vox-points in various neighboring shapes and sizes. Treetop candidates are detected according to the height of the vox-points. In this experiment, five height levels were defined, i.e., 1-5, 5-10, 10-15, 15-20, and $>20 \mathrm{~m}$, which represent the distance between the ground level and the approximate maximum tree height at 5-m intervals. A 1-m buffer zone was added to the ground interval to remove the small bushes near the ground. It should be noticed that the treetops are detected using the weight values of the SEs rather than the weight value of a particular vox-point. This is because the weight values of the SEs represent the distribution of the ALS points in certain neighborhoods of a location, which reflects the shape of an object. By contrast, the weight value of a vox-point represents the ALS point density at a similar location, which does not provide much information on the shape of an object.

The constraints of the treetop detection in this experiment are listed in Table VI. The thresholds are defined considering the possible crown sizes of the trees at different height intervals, the resolution of the voxel space, and the ALS point density of the applied data. The thresholds listed in Table VI are only suitable for the application of the ALS point density, i.e., below $10 \mathrm{pts} / \mathrm{m}^{2}$. In this experiment, the same thresholds were used for the point densities of 2,4 , and $8 \mathrm{pts} / \mathrm{m}^{2}$. A higher ALS point density may provide a richer description of crown shapes, i.e., a single crown may generate more laser returns at different locations. Therefore, higher weight values for SEs can be expected. The thresholds for treetop detection may be adjusted with respect to the ALS point density used.

In a second step, a clustering process is conducted to group the candidate treetops to meet the third morphological characteristic, i.e., that two treetops are not spatially attached. The clustering processing is based on the 3-D distance between the vox-points that are selected as the treetop candidates in the previous step. For each candidate treetop, all other neighboring candidate vox-points that are within a distance $d_{c}$ will be clustered into the same group. The grouping continues until no other candidate can be found within the $d_{c}$ neighborhood of any member in the group. In this paper, $d_{c}$ is $1.5 \mathrm{~m}$, which is an empirical value based on the observation of the minimum 
distance between the treetops in ordinary forest and on the resolution of the voxel space. After the clustering, the treetops can either be a group of vox-points or a single vox-point. For a group of candidate vox-points, the 3-D position of the treetop is calculated from all vox-points. The plane position is the average, and the height is the maximum height of all candidates.

3) Extraction of Individual Tree Crowns: For the detected treetops, the vox-points of their corresponding tree crowns are extracted from the vox-cloud in a bottom-up order, e.g., lower and smaller treetops are given higher priorities, to guarantee the completeness of subordinate trees in a stand.

A cylinder buffer zone surrounding each treetop is calculated to extract the tree crown points. The cylinder has the same height as the treetop. The radius of the cylinder is calculated using a mathematical crown width model for predicting tree crown width according to tree height [24], i.e.,

$$
R_{\text {Org }}=(1.2+0.16 \times H) \times 0.5
$$

where $R_{\text {Org }}$ is the maximum radius of a tree crown, and $H$ is the height of the tree.

All vox-points inside the cylinder are selected and clustered according to the 2-D $(x, y)$ plane distance of the selected points to the treetop at each height level using the following equation:

$$
D_{i}=\overline{d\left(v p_{i}, T\right)}+2 \times s\left(d\left(v p_{i}, T\right)\right)
$$

where $i$ is the tree-level height, $i \in \operatorname{int}(1, h) ; D_{i}$ is the clustering distance at height $i$; $v p_{i}$ is the set of selected vox-points at height $i ; T$ is the treetop; $d$ is the 2-D plane distance; and $s$ is the standard deviation. The first part of the equation is the mean 2-D distance of selected vox-points to the treetop. The second part is the standard deviation of the distances between the vox-points and the treetop. The definition of $D_{i}$ implies $95 \%$ reliability of the selected vox-points in the cylinder buffer zone to be considered as tree crown points. The tree crown cluster is a set of vox-points that satisfy the following condition:

$$
\text { TCrown }=\left\{v p_{i j} \mid d\left(v p_{i}, T\right)<D_{i} ; i \in(1, h) ; j \in\left(1, N_{i}\right)\right\}
$$

where $v p_{i j}$ is the No.j vox-point within the vox-point set $v p_{i}$ at height $I, h$ is the height of the tree, and $N_{i}$ is the total number of selected vox-points at height $i$.

In the final step, all original ALS points that are located in the voxels of a tree crown are extracted, as shown in Fig. 4. The final treetop location and height are defined by the highest ALS point within the tree crown. Other tree crown attributes, namely, the crown height, the crown width, and the crown depth, are also derived from the tree crown cluster of the original ALS points.

\section{RESUlts}

The ING-R, SLU-R, and SLU-H methods were implemented using the data with a point density of $8 \mathrm{pts} / \mathrm{m}^{2}$. The UZH-H and FGI-P methods were used for the three point clouds with point densities of 2,4 , and $8 \mathrm{pts} / \mathrm{m}^{2}$. The results were marked as FGI_2,FGI_4, and FGI_8 for the FGI-P method and UZH_2, UZH_4, and UZH_8 for the UZH-H method. The numbers at the end represent the corresponding point densities of the data

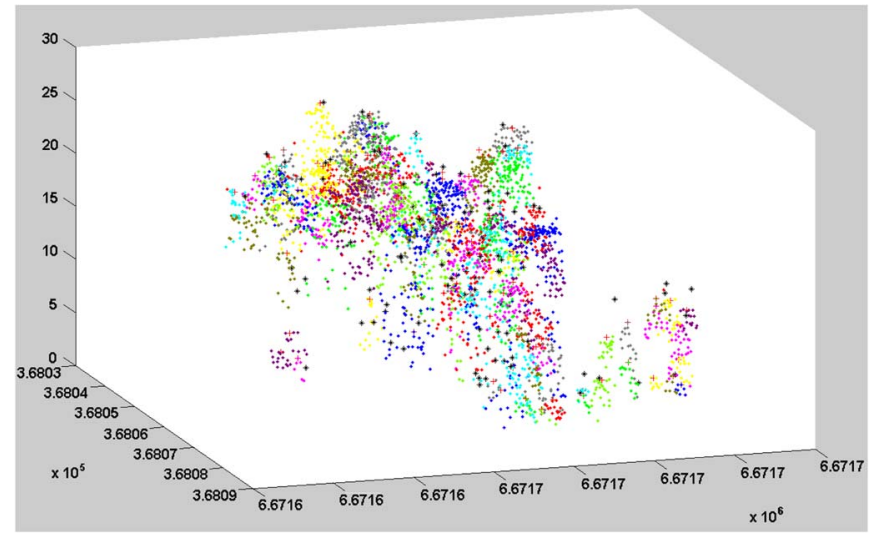

Fig. 4. Extracted individual tree crown points in plot A2. Different colors represent different trees.

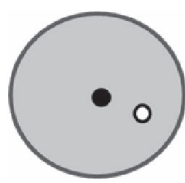

(a)

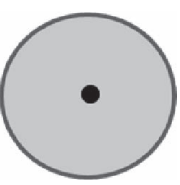

(b)

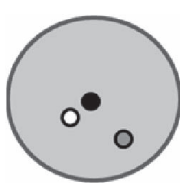

(c)

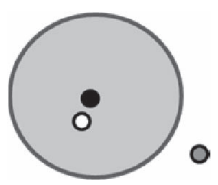

(d)
Fig. 5. Matching cases between reference and detected treetops. Black dots represent the reference treetop. The gray circle surrounding the reference treetop represents a 2-D projection of the buffer spaces in the XY plane. The white dot represents the detected treetop considered as a "match." The gray dots are the commission errors. (a) Match. (b) Omission. (c) Commission I. (d) Commission II.

used. In this section, the tree detection results of all five plots were summed up for the evaluation such that the performance of the new methods in this paper can be compared with the other methods presented in [18].

\section{A. Methods of Evaluation}

The accuracy of detected trees is evaluated according to the 3-D distance between their treetop locations and the nearest reference treetop. The different matching cases between the reference and detected treetops are illustrated in Fig. 5. For the dominant trees (Dom and CoD), correct tree detection, i.e., a "match," means there is one detected treetop within a 3-D spherical buffer space surrounding a reference treetop, and the radius of the sphere is $2.5 \mathrm{~m}$. For the subordinate trees (Int and Sup), the reference buffer space for a "match" is widened to represent a cylinder with a $2.5-\mathrm{m}$ radius and a $3.5-\mathrm{m}$ height because the height of subordinate trees in some cases is difficult to determine with manual reference measurements using TLS. An omission error occurs when a detected treetop cannot be found within the buffer space of a reference treetop. A commission error refers to detected trees that are redundant in the reference data. In this paper, the commission error is divided into two different cases: Commission I refers to an extra treetop detected within the buffer space of a reference treetop; Commission II refers to an extra treetop detected outside the buffer space of any reference treetop, and the crown class of this commission error is allocated according to the nearest reference tree in its 3-D neighborhood. 


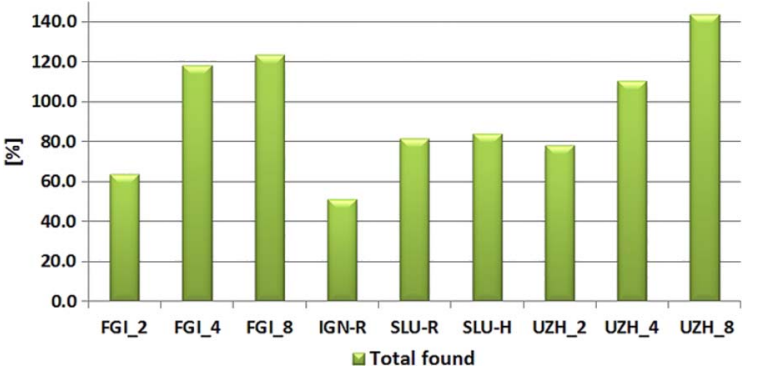

(a)

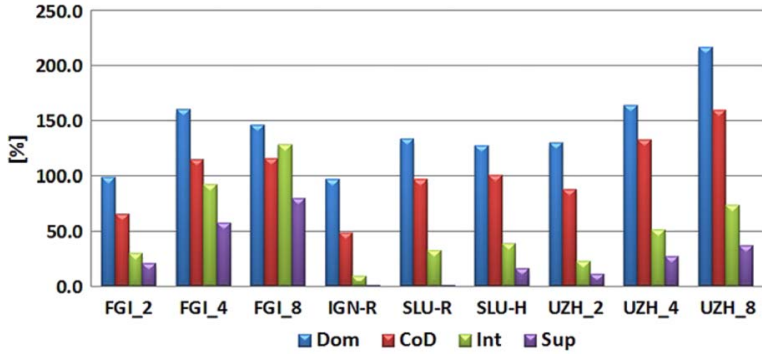

(b)

Fig. 6. Tree detection results of the five methods and the three point densities. A number larger than $100 \%$ means the overestimation of the number of trees,

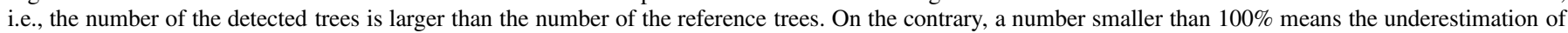
the number of trees. (a) Overall number of detected trees. (b) Crown-class-specific number of detected trees.

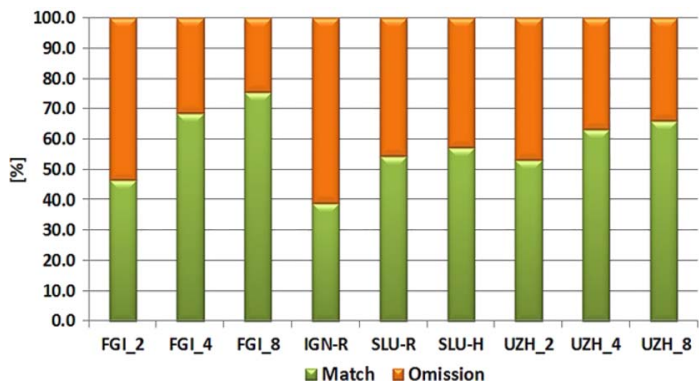

(a)

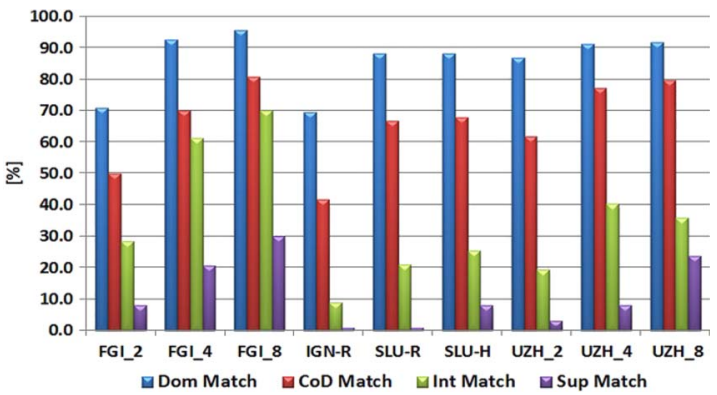

(b)

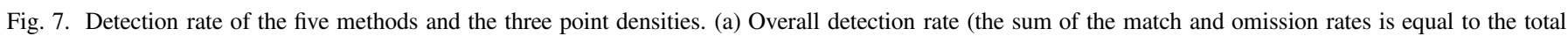
number of reference trees). (b) Crown-class-specific detection rate.

In [18], a "match" was defined using a cylinder buffer space with a 3-m radius and 5-m height with respect to each reference treetop. In this paper, a "match" is defined more strictly because the five methods presented here were recently developed, and they are expected to perform better with respect to tree detection in comparison to the methods previously compared in [18].

\section{B. Number of Detected Trees}

The number of detected trees was evaluated with respect to the total number of reference trees in the test plots. A value of $100 \%$ indicates that the number of detected trees is equal to the number of reference trees when the commission errors are included. The overall and crown-class-specific numbers of detected trees are shown in Fig. 6, which provides an overview of the overestimation and/or underestimation of the number of trees from each method.

In general, the IGN-R, SLU-R, and SLU-H methods tend to underestimate while the FGI-P and UZH-H methods tend to overestimate the total number of trees. Considering the crown class, all methods tend to overestimate the number of dominant trees (Dom and $\mathrm{CoD}$ ). An underestimation of tree number occurred mainly in the subordinate trees (Int and Sup). The raster-based methods (IGN-R and SLU-R) did not detect any trees in the secondary canopy layer (Sup). The utilization of the point cloud (FGI-P, SLU-H, and UZH-H) is shown to be necessary for detecting subordinate trees, particularly for the trees in the secondary layer.

These results reveal that the raster-based methods are insufficient for depicting the 3-D canopy structure of the forest, whereas the point-based and the hybrid methods tend to simplify the canopy structure, particularly in the vertical direction.

All of the compared methods that employ raster models to a certain extent, e.g., IGN-R, SLU-R, SLU-H, and UZH-H, tend to exhibit large differences between the number of detected dominant (Dom and CoD) and subordinate trees (Int and Sup). The point-based method, i.e., FGI-P, provides a more balanced detection of both dominant and subordinate trees, which is important for understanding the developmental stage and canopy structure of a stand. Furthermore, the point density has a clear influence on the performance of the methods that use the point cloud (FGI-P and UZH-H), particularly for the detection of subordinate trees. In general, an increase in the point density improves the tree crown delineation results.

\section{Accuracy of the Methods}

The accuracy of the methods was evaluated considering the detection rate and the rate of commission errors.

The detection rate that represents the proportion of reference trees that were detected is illustrated in Fig. 7. The detection rate also specifies the omission error for a particular method because the sum of the detection rate and the omission rate is always 100\%, as shown in Fig. 7(a). The crown-class-specific detection rate is shown in Fig. 7(b). Almost all methods provide a detection rate of over $85 \%$ for Dom trees and over $70 \%$ for CoD trees. Detection of subordinate trees (Int and Sup) is challenging for all methods. The omission of subordinate trees is the main reason for the low overall detection rate of some methods. 
The utilization of ALS points in tree delineation improves the detection of Int trees and is necessary for the detection of Sup trees. Therefore, only three methods (FGI-P, SLU-H, and $\mathrm{UZH}-\mathrm{H})$ are capable of detecting Sup trees. The best detection rate of the Int $(70.1 \%)$ and Sup $(30.2 \%)$ trees was observed from the FGI-P method using $8 \mathrm{pts} / \mathrm{m}^{2}$ point data. The limitation of ALS for capturing the secondary canopy layer due to obstruction by the topmost canopy layer is one of the reasons for the low detection rate of Sup trees. This is discussed in more detail in Section V-A.

Two methods, i.e., FGI-P and UZH-H, provided detection results using different point densities. There was a clear improvement in the detection rate of Int and Sup trees with an increase in the point density. Kaartinen et al. in [18] concluded that the point density has a small influence on the detection accuracy. This statement needs to be expanded in more detail. For the raster-based methods that only use certain features of selected ALS points, the point density has limited influence on the tree detection accuracy. However, for the methods using ALS points, the density of the point cloud data has a significant influence on the detection rate, particularly for the Int and Sup trees.

The commission error represents the detected trees without any corresponding references. For a clearer understanding of the commission error, two types of commission errors are defined in this study. Commission I errors refer to the extra detection within the 3-D buffer space of a reference treetop, which indicates the tolerance of a method to local height variance surrounding the treetop. A higher Commission I error implies that a method is more sensitive to morphological changes in an individual crown shape in the treetop area. Commission II errors refer to an extra treetop that is detected outside the buffer space of any reference treetop, and the crown class of such an error is allocated according to the nearest reference tree in its 3-D neighborhood. Commission II errors present the sensitivity of a method toward morphological changes, i.e., canopy height variance at the edge or adjunctions of crowns. While Commission I errors usually cause the split of a tree crown along its center, Commission II errors generally occur at the location of large branches or conjunction areas between crowns. The differentiation between these two commission errors helps clarify the challenges of ALS-based ITD considering the tree species and crown classes.

The proportion of the two types of commission errors of each method is shown in Fig. 8. The point-based methods (FGI-P and $\mathrm{UZH}-\mathrm{H}$ ) were shown to have higher commission error rates than the raster-based methods (IGN-R and SLU-R). However, the relatively lower commission rate of the raster-based methods was achieved through the relatively low detection rate, as shown in Fig. 7(a). Further discussion on this issue is presented in Section V-B.

\section{DISCUSSION}

The detection rates of the five algorithms with respect to stand characteristics and crown classes, the cost of the commission errors, and the capability of ALS to measure suppressed trees are discussed in this section. The knowledge derived from this benchmarking reveals the status, potential, and challenges

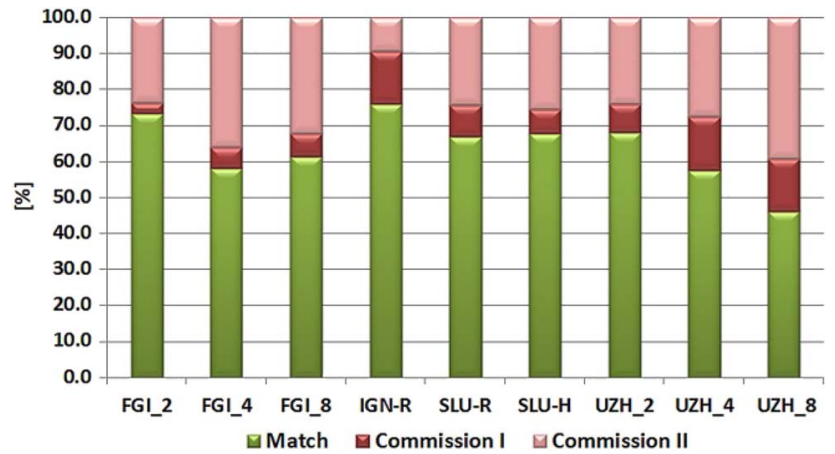

Fig. 8. Commission errors of the various methods. The sum of the match and Commission I and Commission II errors is equal to the total number of trees detected by a particular method.

of using ALS data to measure the 3-D forest canopy structure in large areas.

\section{A. Stand, Crown Class, and Detection Rate}

The detection rate of different crown classes in each test plot was evaluated for an in-depth investigation of the relationship between the stand condition, the crown class, and the detection accuracy. Each crown class was evaluated separately, and the results are illustrated in Fig. 9.

The five forest plots consist of diverse stand conditions and are at different developmental stages, ranging from deciduousdominated (A1) to mixed (A2) to coniferous-dominated (B1-B3) and from old (B1) to mature (A1, A2, B2) to very young (B3) stands. Plot B3 had the lowest detection rate among the five methods (see Fig. 9), which indicates that it is difficult to use ALS-based ITD for the young and dense stands. Once a stand matures and the stem density is less than $500 \mathrm{stem} / \mathrm{ha}$, the detection rate of dominant trees (Dom and CoD) can be achieved at a stable level, i.e., $80 \%-95 \%$ for Dom trees and $70 \%-90 \%$ for $\mathrm{CoD}$ trees, regardless of the species composition in the stands, e.g., deciduous-dominated, coniferousdominated, or mixed. Among the compared methods, FGI-P and UZH-H provide a more robust detection of dominant trees (Dom and CoD) with respect to different tree species and species composition in stands. The SLU-R and SLU-H methods deliver better results for homogeneous/pure stands, irrespective of whether they are dominated by deciduous or coniferous species. The IGN-R method works best for deciduous species regardless of stand condition.

The crown class of trees plays a crucial role in ALS-based ITD, and its influence is even more significant than the tree species. In plots $\mathrm{A} 1$ and $\mathrm{A} 2$, more than $80 \%$ of Dom trees are deciduous, whereas more than $80 \%$ of Dom trees in B1 and B3 are coniferous. In plot B2, 75\% of the Dom trees are coniferous. However, except for plot B3, which is a young and dense stand, the Dom trees in the other four plots had a high detection rate (approximately 90\%) for most of the methods, and there is no obvious difference among the plots. The omission errors for Dom trees mainly originate from the very short trees located in the open areas of a stand, some of which cannot be detected due to the lack of ALS points. 


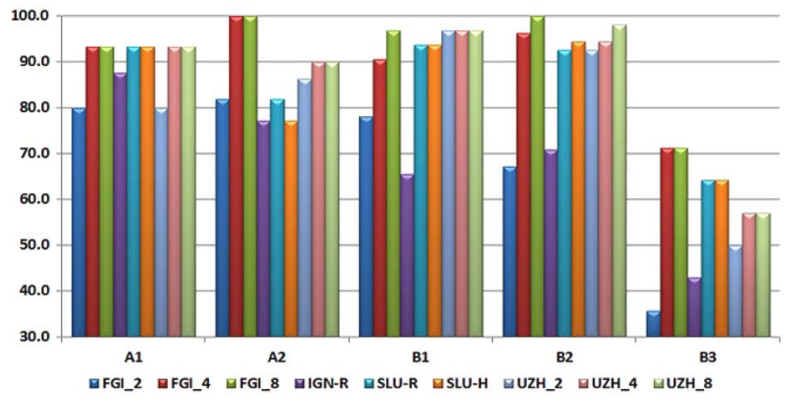

(a)

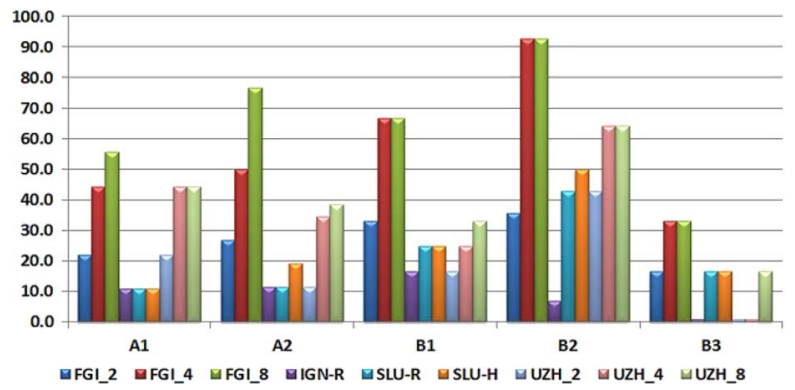

(c)

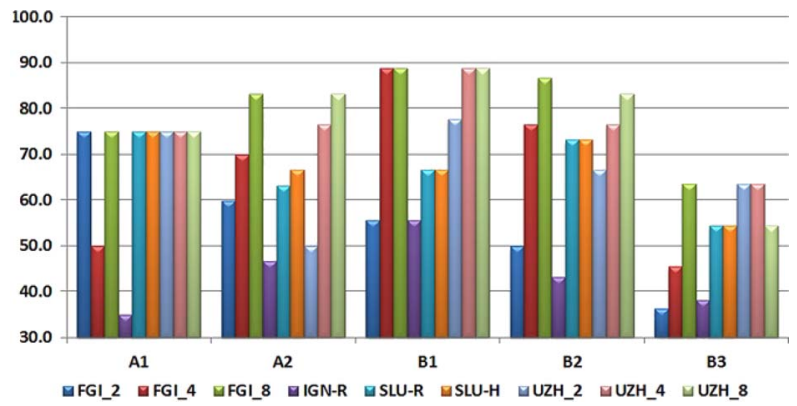

(b)

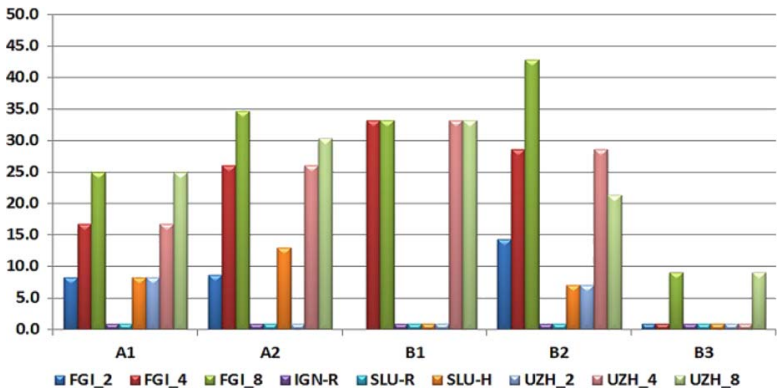

(d)

Fig. 9. Detection rate of different crown classes in each test plot. (a) Dominant trees. (b) Codominant trees. (c) Intermediate trees. (d) Suppressed trees.

A similar situation is observed with the CoD trees. In plots $\mathrm{A} 1, \mathrm{~B} 1$, and $\mathrm{B} 3$, more than $70 \%$ of the CoD trees are coniferous, and $\mathrm{A} 2$ has almost equal numbers of $\mathrm{CoD}$ coniferous and deciduous trees. However, there is no significant difference in the detection rate of the $\mathrm{CoD}$ trees between the four plots. In general, the detection rate of $\mathrm{CoD}$ trees exceeds $70 \%$, and the FGI-P and UZH-H methods have the highest CoD tree detection rates (above 80\%). The homogenous tree height distribution in the neighborhood is the biggest challenge for detecting $\mathrm{CoD}$ trees. The main source of omission errors of $\mathrm{CoD}$ trees is the failure to separate two or three $\mathrm{CoD}$ trees that have similar heights and that are located close to each other.

The detection of subordinate trees (Int and Sup) is more challenging in general. The overall detection rate of Int trees is $40 \%-80 \%$ for the FGI-P and UZH-H methods, and even lower for the other three methods. Based on the results, the detection of deciduous Int trees is more challenging than that of coniferous Int trees. In plot A1, 78\% of the Int trees are deciduous, and this plot had the lowest detection rate among the mature plots (A1, A2, B1, and B2) when the FGI-P, SLU-R and SLU-H methods were used. The height of the Int trees also influences the detection rate. In the stands where the average height of the Int trees is approximately $10 \mathrm{~m}(\mathrm{~A} 2$ and $\mathrm{B} 2)$, the detection rate of the FGI-P and UZH-H methods is higher. However, species and height are not the primary factors that determine the detection of Int trees. The most critical influencing factor is the canopy morphology in the neighborhood of the Int trees. The possibility of an Int tree being correctly detected is decided by the crown shape of the neighboring dominant (Dom and $\mathrm{CoD}$ ) trees as well as by the height difference between the Int tree and its neighboring dominant trees. The clearer the height variance between the different canopy classes in a stand (e.g., plots A2 and B2), the easier it is to detect Int trees.
Trees in the secondary canopy layer, i.e., Sup trees, had the lowest detection rate. One major reason is the limited number of ALS points reflected from the Sup trees. The capability of ALS to capture suppressed trees is discussed in detail in Section V-C. Similar to the Int trees, the correct detection of a Sup tree is highly influenced by the species and the height and crown shape of its neighboring trees rather than by the species and the height of the Sup tree itself. Different methods have varied performances under different stand conditions. SLU-H works better for the higher Sup trees. Both FGI-P and UZH-H perform better under conditions where the height variance between the Sup trees and their neighboring trees is high. The height of a Sup tree itself does not have a significant influence on the detection result of these two methods (FGI-P and UZH-H). The two raster-based methods (IGN-R and SLU-R) do not provide information on Sup trees.

\section{B. Cost of Commission Errors}

1) Two Commission Cases: The commission errors of the compared methods for different crown classes in each test plot are illustrated in Fig. 10. The results show that the number of Commission I errors is strongly related to the tree species, particularly for the dominant trees (Dom and CoD). For all the compared methods, a high rate of Commission I errors can be found for dominant trees in plots $\mathrm{A} 1$ and $\mathrm{A} 2$ where the Dom and $\mathrm{CoD}$ trees are mainly deciduous. The Commission I error of the Dom and CoD trees is clearly lower in the coniferous stands (B1-B3).

The case for the Commission II error is, however, quite different. The Commission II errors are maintained at a similar level for both coniferous and deciduous Dom trees. A similar 

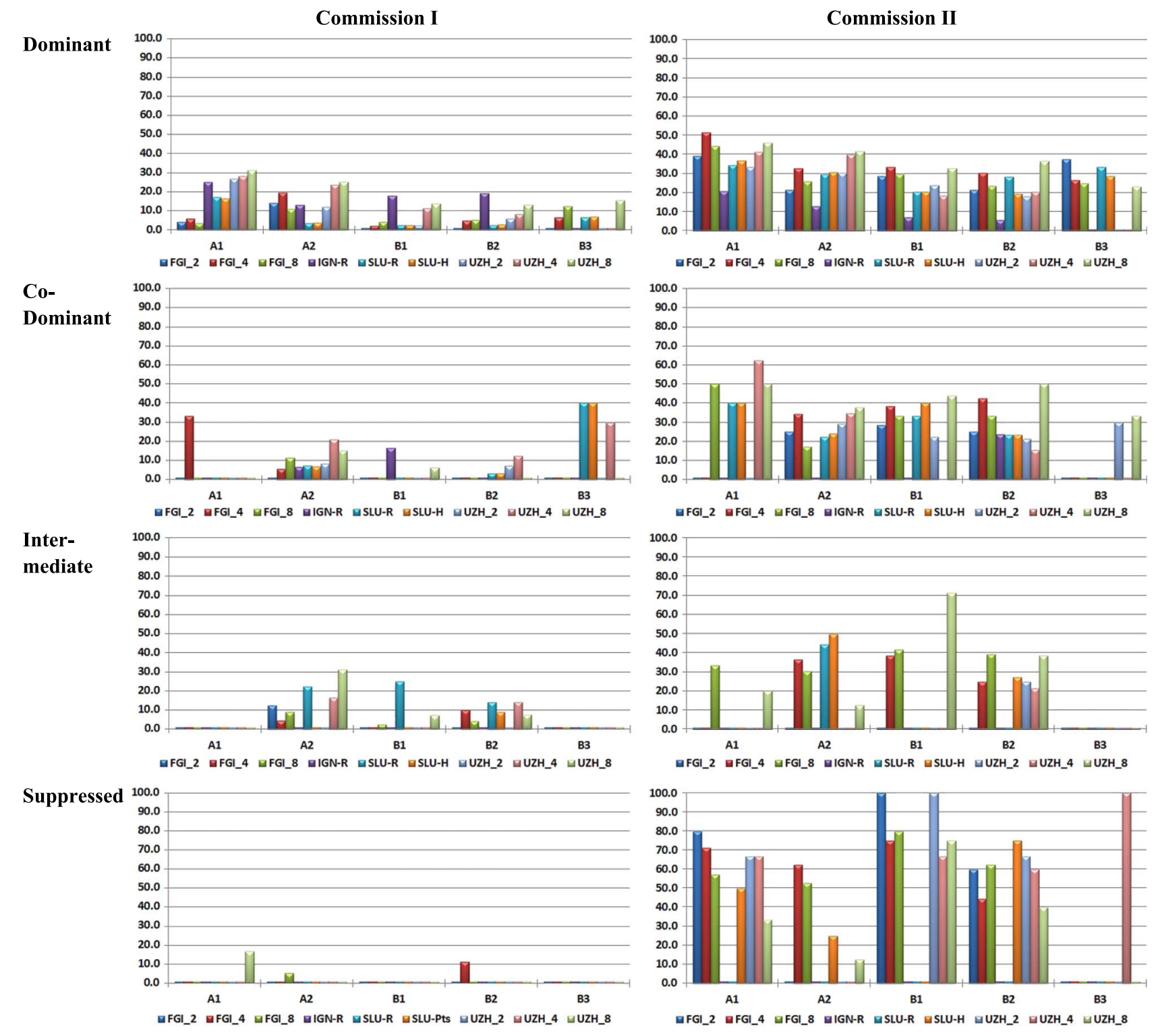

Fig. 10. Commission errors of different crown classes in each test plot.

result is observed for the CoD trees. This implies that the height variance at the edge of a large crown and/or the conjunction area of neighboring crowns leads to a high risk of Commission II errors, which presents the biggest challenge for the detection of dominant trees (Dom and CoD), and such challenges are not species or stand specific.

The detection of subordinate trees (Int and Sup) is a challenging task for all the compared methods. As previously mentioned, the species and heights of the subordinate trees alone do not explain their detection accuracy levels. The distribution of commission errors among the Int trees also reflects this fact. Plots $\mathrm{A} 2, \mathrm{~B} 1$, and $\mathrm{B} 2$ have higher commission errors (I and II) with respect to Int trees, but the Int trees in these three plots are mainly coniferous. The influencing factor for with a greater impact on the detection accuracy of subordinate trees is the complexity of the local 3-D canopy structure, i.e., the distribution of the crown classes in the neighborhood.
Another important fact associated with the detection of Int trees is the balance between a high detection rate and a large number of commission errors. Detecting Int trees requires a method to be sensitive toward the local height variance of the canopy such that the lower treetops of Int trees that are suppressed by the neighboring dominant trees (Dom and CoD) can be detected. However, large branches of dominant trees as well as the conjunctions of dominant tree crowns may present morphological features of the canopy structure that are similar to those of intermediate trees and their surrounding Dom and $\mathrm{CoD}$ trees. Both situations consist of a recognizable height variance of the individual crown or crown group. Efforts to detect the treetops of Int trees will therefore simultaneously increase the Commission II error associated with the large branches or the conjunction area of Dom and CoD trees. In brief, the increase in commission error, particularly Commission II error, is a side effect of pursuing a high detection rate of Int trees, which is very challenging for all ITD methods. 


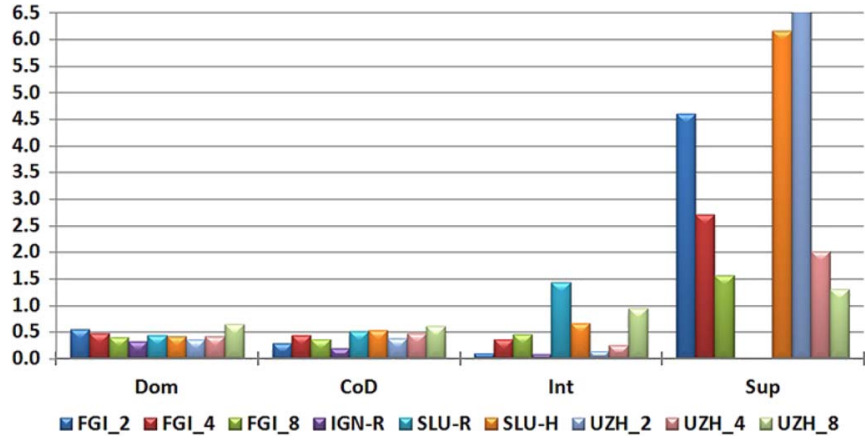

Fig. 11. Com_Cost factor for each method for different crown classes.

For the suppressed trees, the Commission II error resulting from the FGI-P and UZH-H methods is high, which significantly contributes to the overall commission error rate of these two methods (see Fig. 8). One reason could be that the shrubs and bushes that are not included in the reference data have been detected by the methods. Another source of such commission error may be the large spruce or pine branches located at low heights in the stand. The result also shows that an increase in the point density reduces the Commission II errors for suppressed trees. This is because a higher point density will record the treetops of suppressed trees in more detail, and it is easier to differentiate these canopies from the other points on low branches.

2) Match-Commission Tradeoffs of the Methods: A comparison between the correct detections and the commission errors of the five methods shows that the commission error is positively correlated with the detection rate. A higher overall detection rate tends to result in a higher commission error. This indicates that the high tree-detection rate most probably increases the commission error, and addressing the tradeoff between "match" and "commission" is one of the main challenges for ITD. One technique to assess the tradeoff between "match" and "commission" is to estimate the stem volume distribution for both cases and to evaluate the extent of the stem volume overestimation caused by the commission errors. However, the estimation of stem volume itself can be biased considering different species and stand conditions.

In this paper, the commission error is considered as a cost of correct detection, and each method has different costs of commission errors associated with the correct detection. The measurement of the match-commission tradeoff is evaluated using the commission cost, which is defined by the following equation:

\section{Com Cost $=$ Commission Rate/Detection Rate}

The Com_Cost factor represents the cost of commission error (including both Commission I and II errors) per each percentage of the detection rate. A low Com_Cost factor for a method means the method can produce its detection rate with a low rate of commission error, which is an indication of a proper balance between accurate detection and commission error. The Com_Cost factor for each method for different crown classes in all five plots is illustrated in Fig. 11.

Almost all methods had a low Com_Cost factor (below 0.5) for the Dom and CoD trees, which shows that the performance of the compared methods in controlling the commission errors associated with the dominant trees is plausible. However, it should be noted that the Com_Cost factor implies a balance between accurate detection and the commission errors; the factor alone does not represent the efficiency of a method. A low Com_Cost factor may be associated with a low detection rate. A good balance of the tradeoff between the correct detection and the commission errors is to obtain a high detection rate (e.g., above $80 \%$ ) with a low Com_Cost factor (e.g., below $0.5)$. In this respect, the FGI-P and SLU-H methods provide the most satisfactory results. The IGN-R method has the lowest Com_Cost factor for the Dom and CoD trees, as well as the lowest detection rate. On the contrary, the UZH-H method has a high detection rate and a high Com_Cost factor.

The appearance of the commission errors associated with the Int trees is highly relevant for the detection of these Int trees. The SLU-R, SLU-H, and UZH-H Com_Cost factors for the Int trees are clearly higher than those of the Dom and CoD trees. The reason for this might be that these three methods rely on rasterized data (images) for detecting Int trees, and the balancing of the match-commission tradeoffs is notably difficult for the raster-based methods. The utilization of point clouds may reduce the Com_Cost factor, as shown by the difference between the SLU-R and SLU-H methods.

The Com_Cost factor associated with the Sup trees was not evaluated for IGN-R and SLU-R because these methods do not detect suppressed trees. For the other three methods, the Com_Cost factor was much higher for the Sup trees compared with the other crown classes. As previously discussed, one reason for the high commission error is the absence of shrubs and bushes in the reference data. On the other hand, an increase in the point density obviously reduces the Sup tree Com_Cost factor, which confirms the importance of a high point density for the detection of subordinate trees at the secondary canopy layer.

This study of the Com_Cost factor indicated that: 1) to pursue a high detection rate while maintaining a low commission error is an achievable task for the dominant trees (Dom and $\mathrm{CoD}$ ), but it is challenging for the subordinate trees (Int and Sup);2) the utilization of point cloud data in the ITD methods is important in dealing with the tradeoffs between the detection rate and the commission errors; and 3) a higher point density clearly benefits the reduction of the Com_Cost factor for detecting the Sup trees.

\section{Capability of ALS to Detect Suppressed Trees}

The penetration of forest canopies by laser beams is one of the most competitive characteristics of ALS, which makes it possible to study the vertical canopy structure and the undergrowth of the secondary canopy layer using remote sensing technology over large areas. The features that are related to the scanning system (e.g., discrete or full-waveform, the pulse frequency, etc.) and the flight (e.g., flight height, scanning angle, etc.) are assumed to have a direct influence on the ability of ALS to capture the secondary canopy layer. Forest canopy characteristics, e.g., canopy closure and leaf area, also influence the capturing capabilities. Until now, there has been a lack of 
TABLE VII

Number of Detectable Suppressed Trees in Each Test Plot

\begin{tabular}{lcccccc}
\hline & A1 & A2 & B1 & B2 & B3 & Average \\
\hline Total Suppressed Trees & 12 & 23 & 3 & 14 & 11 & \\
Detectable Suppressed Trees & 4 & 10 & 1 & 7 & 1 & \\
Detectable Rate (\%) & 33.3 & 43.5 & 33.3 & 50 & 9.1 & 33.9 \\
\hline
\end{tabular}

quantifiable evaluations of the efficiency of the ALS data to capture the 3-D canopy structure, particularly the Sup trees that are often occluded by other canopy layers.

The number of detectable Sup trees in the applied first-last return ALS data $\left(8 \mathrm{pts} / \mathrm{m}^{2}\right)$ is evaluated in this study. A Sup tree is considered as "detectable" using automated methods when there are a certain number of ALS points that describe its crown. Three methods in this study (FGI-P, SLU-H, and $\mathrm{UZH}-\mathrm{H})$ require a cluster of points at the crown area for the detection of Sup trees. Considering the ALS point density as well as the criterion of the "point cluster" in the three methods, the definition of "detectable" is set to at least 5 points within a cylinder space with a 2.5 -m radius and 3-m height surrounding the treetop of a reference Sup tree. To mitigate the possible height bias in the reference data, the top of the cylinder space is set to $1 \mathrm{~m}$ higher than the reference treetop.

The number of detectable Sup trees that satisfy this definition and the corresponding total number of Sup trees in the reference data are presented in Table VII. Plots A2 and B2 have a relatively higher rate of detectable Sup trees, most probably because there is a more homogenous distribution of different species as well as crown classes in these two stands. The dominant populations of deciduous trees (A1) and old coniferous trees (B1) in the topmost canopy layer may reduce the detectable rate of the Sup trees in the stands due to higher leaf area and/or canopy closure. The possibility of detecting Sup trees in the young and dense stand (B3) is even lower, partly because of the lack of a clear canopy structure in this stand. In B3, the high stem density is another challenge for the ALS to detect the Sup trees.

The average detectable rate of the Sup trees in the five test plots is $33.9 \%$, which indicates that the first-last return ALS data with $8 \mathrm{pts} / \mathrm{m}^{2}$ point density is capable of capturing approximately one-third of the Sup trees in stands. This fact implies that the performance of the automated methods for detecting Sup trees, as shown in Fig. 9(d), is plausible. The actual detection rate of the Sup trees, calculated from the detectable number rather than the total reference number, can reach up to $88.2 \%$ for the FGI-P method, $37.7 \%$ for the SLU-H method, and $63.1 \%$ for the UZH-H method.

It should be emphasized that the overall detectable rate of Sup trees is only evaluated for the applied ALS data that were collected more than ten years ago by repeated flight lines to achieve higher point densities. With the rapid progress of scanner technology, new data usually have the ability to provide more intermediate returns and a higher point density, which should improve the capability of capturing the Sup trees in stands. It is a pity that test areas have been felled during the last ten years, and it is impossible to obtain new data for the same test plots because it would be interesting to compare the difference resulting from the different scanning systems.

\section{CONCLUSION}

This paper has clarified the current status of the ITD approaches with respect to their capabilities to depict 3-D canopy structures through international benchmarking. Five ITD methods, i.e., two raster-based (IGN-R and SLU-R), two hybrid (SLU-H and UZH-H), and one point-based (FGI-P), were evaluated and compared, in which the point-based method (FGI-P) is a newly developed approach introduced in this paper. The methods were compared using three ALS data sets with different point densities $\left(2,4\right.$, and $\left.8 \mathrm{pts} / \mathrm{m}^{2}\right)$ collected in five boreal forests with diverse stands at different development stages.

The capability of ITD methods to depict the 3-D canopy structure was evaluated at a crown-class level. Individual crown classes were evaluated separately, and detailed analyses of the detection accuracy of subordinate trees, i.e., the intermediate and suppressed trees, are reported for the first time through benchmarking in this study. In addition, the challenges of modeling 3-D canopy structure using ITD approaches are investigated from different perspectives, i.e., the methodology, the stand situation, and the quality of the applied ALS data.

It is revealed that, at the current stage, the raster-based ITD methods are shown insufficient to depict the 3-D canopy structure due to their incapability to detect trees in the secondary canopy layer. The point-based and hybrid methods tend to simplify the canopy structure, particularly in the vertical direction, since the number of subordinate trees is typically underestimated.

With respect to the methodology, this study reveals the importance of utilizing the point cloud data in the ITD methods, which is necessary for depicting the 3-D canopy structure, and benefit of dealing with the challenges of commission errors, particularly when high detection rates for codominant and intermediate trees are expected. Clear tradeoffs are observed between the detection rate and the commission errors. The commission errors that occur at the edge of the crown or the conjunction area between crowns are more challenging, because the large branches as well as the conjunction areas of dominant and codominant trees may present morphological features in the crown structure that are similar to those of the intermediate trees, which means that any attempt to detect intermediate trees simultaneously increases the risk of generating commission errors. It is difficult for the raster-based methods to differentiate the large separated branches or certain conjunctions of neighboring crowns from the real intermediate trees because information of vertical dimension is typically lost in the rasterized data. The application of the point cloud data can mitigate such a challenge by taking more vertical information into consideration, such as the crown depth and the crown cluster size.

Considering the impacts of the stand structure, this benchmarking study also revealed that the crown class plays a considerable role in the detection accuracy of ITD methods, and its influence is even greater than that of the tree species as well as the species composition in a stand. In mature stands, the overall detection rate of dominant trees can exceed $90 \%$, and that of codominant trees can exceed $80 \%$ for most of the methods, regardless of the tree species and the species composition in the stand. The canopy structure has a greater impact on the detection rate of the subordinate trees, i.e., intermediate and suppressed trees, than the species and height of the subordinate 
trees themselves. The detection rate of the intermediate trees ranged from $40 \%$ to $80 \%$ and was even lower for the suppressed trees (up to $30 \%$ ). The clearer the height variance among different crown classes in a stand, the easier it is to detect subordinate trees.

Apart from the methodology and the stand structure, the low detection rate for subordinate tree is partly because only limited laser beams were reflected from the forest undergrowth due to the occlusion caused by dominant trees or the low point density. This study conducted the first quantifiable evaluation of the efficiency of the ALS data to capture suppressed trees. In the $8 \mathrm{pts} / \mathrm{m}^{2}$ ALS data, only $34 \%$ of the suppressed trees in the test area were sufficiently recorded in the ALS point cloud to describe the existence of their canopy. Among the recorded suppressed trees, up to $88 \%$ of the suppressed trees can be detected and modeled. These statistics indicated that the automated ITD methods perform well in detecting suppressed trees in various forest structures; however, at this point density, ALS has limited capability to capture suppressed trees, and the depiction of suppressed trees directly depends on the capability of recording such trees.

Another important finding is that the increase in point density is clearly helpful in the detection of subordinate trees. This is different from what was concluded in [18], where the point density was reported to have limited influence. While being insignificant for the raster-based methods, the point density is a highly influential factor in the performance of the methods that use the point cloud data. In the point-based (FGI-P) and hybrid (UZH-H) methods, an increased point density results in a higher detection rate and a lower cost of commission errors for both intermediate and suppressed trees. On the other hand, the dominant trees were shown to be well depicted in ALS data with all point densities. This finding shows that if the focus of an application is to measure the dominant trees only, ALS data of a point density of 2 points $/ \mathrm{m}^{2}$ may already provide satisfactory results.

With the use of ITD methods that are able to take advantage of the richer information produced by the high-density point clouds, utilization of more advanced multireturn and full-waveform data with higher point density is anticipated to improve the characterization of the 3-D canopy structure by more accurately detecting intermediate and suppressed trees in stands. Again, the application of more advanced ALS systems is only meaningful for the methods that utilize the point cloud data, which indicates that greater effort should be placed on developing and improving the point-based and hybrid ITD methods to model the 3-D canopy structure and to further explore the potential of high-density ALS data.

Measuring 3-D forest structure from ALS has great practical potentials. Applications that use remote sensing data to produce forest attribute maps often require plot-level field data for constructing mathematical models and validating outcomes. Typically, representative field plots are costly to install and, as a result, are often limited in number and extent. As an alternative, Wulder et al. in [5] proposed the concept of the ALS (LiDAR) plot. In such a concept, it is particularly necessary to be able to characterize the subordinate trees, i.e., the intermediate and suppressed trees. While recent ALS systems are capable of providing a very high point density, i.e., above $100 \mathrm{pts} / \mathrm{m}^{2}$, the advantages of such high point density for ALS-based ITD still need to be explored. On the other hand, an extremely high point density does not necessarily benefit the performance of ALS-based ITD considering the usefulness of the points and the computational costs. The marginal utility of point density for ITD performance requires more in-depth study in the future.

Furthermore, ALS systems are adopting wavelengths other than the near-infrared. The Optech Titan ALTM system provides up to three active channels of different wavelengths, which is assumed to be helpful for the classification of species and may consequently benefit ALS-based ITD with respect to higher accuracy. As the application of ALS data expands to more widespread applications in silviculture and forest ecology, both the horizontal and vertical structures of forest canopies are expected to be investigated for the purpose of monitoring regeneration, estimating biomass, or classifying habitats. Our future studies will focus on employing the latest ALS data to improve the study of 3-D canopy structures, ITD in different crown classes, and other parameter estimates for the trees in both the topmost and secondary canopy layers.

\section{ACKNOWLEDGMENT}

The authors would like to thank the European Spatial Data Research Organization for providing the test data.

\section{REFERENCES}

[1] J. Norman and G. Campbell, "Canopy structure," inPlant Physiological Ecology, R. Pearcy, J. Ehleringer, H. Mooney, and P. Rundel, Eds. New York, NY, USA: Springer-Verlag, 1989, pp. 301-325.

[2] G. Kraft,Beiträge zur Lehre von den Durchforstungen, Schlagstellungen und Lichtungshieben. Hannover, Germany: Klindsworth's Verlag, 1884

[3] D. Bayer, S. Seifert, and H. Pretzsch, "Structural crown propertie of Norway spruce (Picea abies [L.] Karst.) and European beech (Fagus sylvatica [L.]) in mixed versus pure stands revealed by terrestrial laser scanning," Trees, vol. 27, no. 4, pp. 1035-1047, 2013.

[4] M. A. Lefsky, W. B. Cohen, G. G. Parker, and D. J. Harding, "Lidar remote sensing for ecosystem studies lidar, an emerging remote sensing technology that directly measures the 3-D distribution of plant canopies, can accurately estimate vegetation structural attributes and should be of particular interest to forest, landscape, and global ecologists," BioScience, vol. 52, no. 1, pp. 19-30, 2002.

[5] M. A. Wulder et al., "Lidar sampling for large-area forest characterization: A review," Remote Sens. Environ., vol. 121, pp. 196-209, Jun. 2012.

[6] J. Hyyppä, M. Holopainen, and H. Olsson, "Laser scanning in forests," Remote Sens., vol. 4, no. 10, pp. 2919-2922, Sep. 2012.

[7] B. Koch, U. Heyder, and H. Weinacker, "Detection of individual tree crowns in airborne lidar data," Photogramm. Eng. Remote Sens., vol. 72, no. 4, pp. 357-363, Apr. 2006.

[8] Y. Wang, H. Weinacker, and B. Koch, "A lidar point cloud based procedure for vertical canopy structure analysis and 3-D single tree modeling in forest," Sensors, vol. 8, no. 6, pp. 3938-3951, Jun. 2008.

[9] A. Persson, J. Holmgren, and U. Söderman, "Detecting and measuring individual trees using an airborne laser scanner," Photogramm. Eng. Remote Sens., vol. 68, no. 9, pp. 925-932, Sep. 2002.

[10] S. C. Popescu, "Estimating biomass of individual pine trees using airborne lidar," Biomass Bioenergy, vol. 31, no. 9, pp. 646-655, Sep. 2007.

[11] X. Yu, J. Hyyppä, M. Vastaranta, M. Holopainen, and R. Viitala, "Predicting individual tree attributes from airborne laser point clouds based on the random forests technique," ISPRS J. Photogramm. Remote Sens., vol. 66, no. 1, pp. 28-37, Jan. 2011.

[12] J. Hyyppä et al., "Advances in forest inventory using airborne laser scanning," Remote Sens., vol. 4, no. 5, pp. 1190-1207, May 2012.

[13] A. Ferraz et al., "3-D mapping of a multi-layered Mediterranean forest using ALS data," Remote Sens. Environ., vol. 121, pp. 210-223, Jun. 2012.

[14] E. Lindberg, L. Eysn, M. Hollaus, J. Holmgren, and N. Pfeifer, "Delineation of tree crowns and tree species classification from full-waveform air- 
borne laser scanning data using 3-D ellipsoidal clustering," IEEE J. Sel. Top. Appl. Earth Observ. Remote Sens., vol. 7, no. 7, pp. 3174-3181, Jul. 2014

[15] X. Liang, V. Kankare, X. Yu, J. Hyyppa, and M. Holopainen, "Automated stem curve measurement using terrestrial laser scanning," IEEE Trans. Geosci. Remote Sens., vol. 52, no. 3, pp. 1739-1748, Mar. 2014.

[16] B. Koch, T. Kattenborn, C. Straub, and J. Vauhkonen, "Segmentation of forest to tree objects," in Forestry Applications of Airborne Laser Scanning. Dordrecht, The Netherlands: Springer-Verlag, 2014, pp. 89-112.

[17] H. Kaartinen and J. Hyyppä, "EuroSDR/ISPRS Project, Commission II 'Tree extraction' Final Report," in Proc. EuroSDR, 2008, pp. 1-60.

[18] H. Kaartinen et al., "An international comparison of individual tree detection and extraction using airborne laser scanning," Remote Sens., vol. 4, no. 4, pp. 950-974, Mar. 2012.

[19] J. Vauhkonen et al., "Comparative testing of single-tree detection algorithms under different types of forest," Forestry, vol. 85, no. 1, pp. 27-40, Oct. 2011.

[20] L. Eysn et al., "A benchmark of lidar-based single tree detection methods using heterogeneous forest data from the alpine space," Forests, vol. 6, no. 5, pp. 1721-1747, May 2015.

[21] Y. Qin, A. Ferraz, C. Mallet, and C. Iovan, "Individual tree segmentation over large areas using airborne LiDAR point cloud and very high resolution optical imagery," in Proc. IEEE IGARSS, 2014, pp. 800-803.

[22] J. Holmgren and E. Lindberg, "Tree crown segmentation based on a geometric tree crown model for prediction of forest variables," Can. J. Remote Sens., vol. 39, pp. S86-S98, 2013.

[23] F. Morsdorf et al., "LIDAR-based geometric reconstruction of boreal type forest stands at single tree level for forest and wildland fire management," Remote Sens. Environ., vol. 92, no. 3, pp. 353-362, Aug. 2004.

[24] X. Liang, J. Hyyppä, and L. Matikainen, "Deciduous-coniferous tree classification using difference between first and last pulse laser signatures," Int. Archives Photogramm., Remote Sens. Spatial Inf. Sci., vol. 36, Part 3/W52, pp. 253-257, 2007.

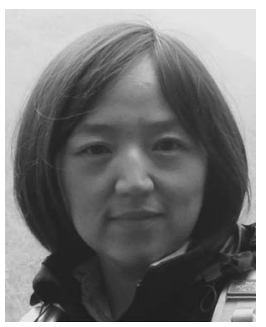

Yunsheng Wang received the Ph.D. degree from the University of Freiburg, Freiburg im Breisgau, Germany, in 2008.

From 2010 to 2012, she was a Project Manager in Environmental Management and Climate Change with the Deutsche Gesellschaft für Internationale Zusammenarbeit (GIZ) GmbH. She is currently a Senior Research Scientist with the Centre of Excellence in Laser Scanning Research, Academy of Finland, Helsinki, Finland, and with the Finnish Geospatial Research Institute, Masala, Finland. Her main research interests lie in the assessment of ecosystem services and 3-D modeling from point cloud data.

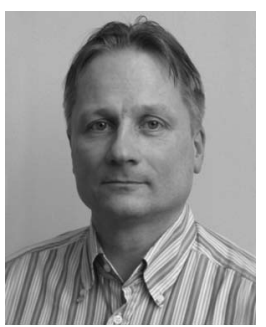

Juha Hyyppä received the M.Sc., Dr.Ing., and Dr.Sc. degrees (with honors) from Helsinki University of Technology, Espoo, Finland, in 1987, 1990, and 1994, respectively.

$\mathrm{He}$ is currently a Professor of remote sensing and photogrammetry with the Finnish Geospatial Research Institute, a Director of the Centre of Excellence in Laser Scanning Research, and a Distinguished Professor with Shinshu University (Japan). His references include 25 years of experience in research team leadership, coordination of over ten international science projects, and author of over 150 ISI Web of Science listed papers. His research is focused on laser scanning systems, their performance, and new applications, related to mobile, personal, and ubiquitous laser scanning and their point cloud processing particularly to forest information extraction.

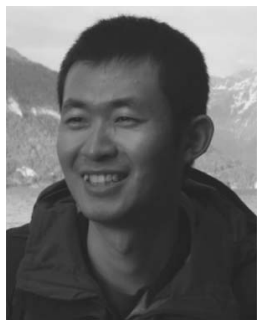

Xinlian Liang received the Ph.D. degree from Aalto University, Espoo, Finland, in 2013.

$\mathrm{He}$ is a Senior Research Scientist and the leader of the remote sensing of forest group with the Department of Remote Sensing and Photogrammetry, Finnish Geospatial Research Institute, Masala, Finland, and with the Centre of Excellence in Laser Scanning Research, Academy of Finland, Helsinki, Finland. His current research interests include innovative geospatial techniques in modeling forest ecosystems, from all kinds of point clouds as well

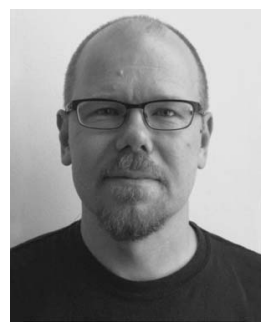

Harri Kaartinen received the Ph.D. degree, with a thesis on benchmarking of laser scanning systems and point cloud processing, from Aalto University, Espoo, Finland, in 2013.

In 1996, he joined the Finnish Geodetic Institute, currently Finnish Geospatial Research Institute, Masala, India, where he is a Research Professor with the Department of Remote Sensing and Photogrammetry. His research interests include laser scanning system performance and new platforms and applications.

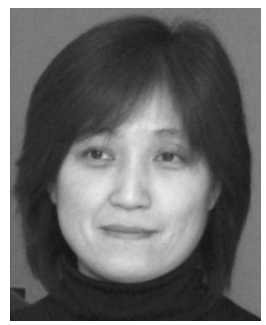

Xiaowei Yu received the Dr.Sc. degree from Aalto University, Espoo, Finland, in 2008.

She is a Specialist Research Scientist with the Department of Remote Sensing and Photogrammetry, Finnish Geospatial Research Institute, Masala, Finland, and with the Centre of Excellence in Laser Scanning Research, Academy of Finland, Helsinki, Finland. Her current research interests include the development of algorithms and methods for laser scanning data processing and information extraction and the applications of laser scanning in forest change detection and forest inventories.

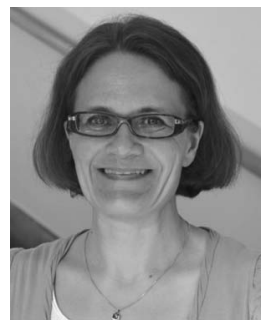

Eva Lindberg received the Ph.D. degree, with a thesis on laser scanning for estimation of canopy structure and individual trees, from the Swedish University of Agricultural Sciences, Uppsala, Sweden, in 2012.

She is currently a Postdoctoral Researcher with the Forest Remote Sensing Section, Department of Forest Resources, Swedish University of Agricultural Sciences. Her research includes applications of laser scanning and other remote sensing techniques for habitat analysis and derivation of novel information from laser scanning for vegetation mapping.

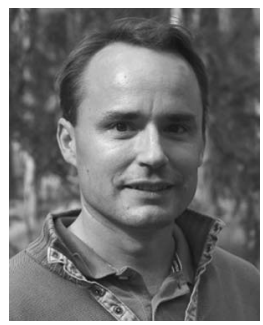

Johan Holmgren received the Ph.D. degree, with a thesis on airborne laser scanning for forestry applications, from the Swedish University of Agricultural Sciences, Uppsala, Sweden, in 2003.

$\mathrm{He}$ is currently a Senior Research Scientist with the Forest Remote Sensing Section, Department of Forest Resources, Swedish University of Agricultural Sciences. His research includes laser scanning and sensor data fusion for forestry applications.

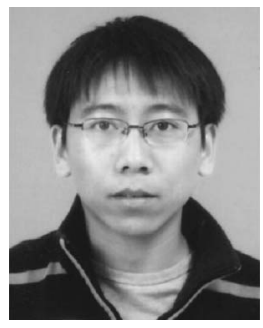

Yuchu Qin was born in Yichang, China. He received the B.S. degree in environment and urban planning from Shenyang Jianzhu University, Shenyang, China, in 2004 and the Ph.D. degree in geodesy and geoinformatics from the Chinese Academy of Sciences, Beijing, China, and the Royal Institute of Technology (KTH), Stockholm, Sweden, in 2010 and 2014, respectively.

From 2011 to 2016, he worked as a Postdoctoral Researcher with South Dakota State University, Brookings, SD, USA; MATIS, IGN, Paris, France; and the Alberta Terrestrial Imaging Center, Canada. His research interests include algorithm development for optical image and LiDAR data processing, photogrammetric computer vision and machine learning, geospatial data integration, and analysis and visualization for operational mapping in forest and urban areas. 


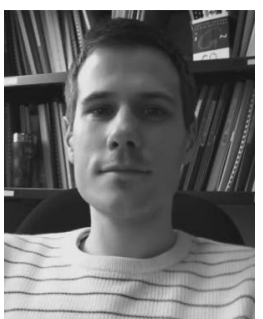

Clément Mallet received the M.Eng. degree from the Ecole Nationale des Sciences Géo-graphiques, Marne-la-Vallée, France; the M.Sc. degree in physics in remote sensing from the Université Pierre et Marie Curie-Paris 6, Paris, France, in 2005; and the Ph.D. degree in image and signal processing from Telecom ParisTech, Paris, in 2010.

$\mathrm{He}$ is currently a Senior Researcher with the French National Institute for Geographic Information and Forestry, Paris. His research interests include point cloud and optical image processing, land-cover classification, and, more generally, multimodal remote sensing.

Dr. Mallet serves as the Chair of the International Society for Photogrammetry and Remote Sensing Working Group on Image Sequence Analysis for 2012-2016. He served as the Editor-in-Chief for the French Journal of Photogrammetry and Remote Sensing between 2011 and 2015.

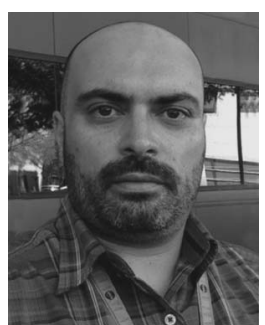

António Ferraz was born in Antas de Penedono, Portugal. He received the M.S. degree in geomatics engineering from Universidade de Coimbra, Coimbra, Portugal, in 2007 and the Ph.D. degree in geophysics from the Institute de Physique du Globe de Paris, Paris, France, in 2012.

From November 2007 to January 2014, he was with the French National Institute for Geographic Information and Forestry, Paris. Since May 2014, he has been with the NASA Postdoctoral Program at the Jet Propulsion Laboratory, California Institute of Technology, Pasadena, CA, USA. His main research interests include remote sensing of vegetation, optical image and 3-D point cloud processing, direct retrieval of forest variables using airborne laser scanning systems, forest 3-D modeling, and large-area carbon and biomass estimation over tropical environments using airborne and satellite data.

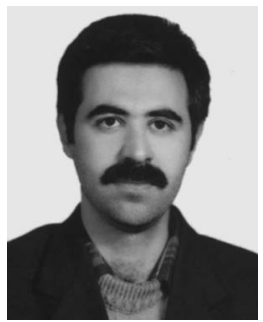

Hossein Torabzadeh received the Ph.D. degree in remote sensing from the University of Zürich, Zürich, Switzerland, in 2015.

$\mathrm{He}$ is currently an Assistant Professor with the Department of Civil Engineering, Bu-Ali Sina University, Hamedan, Iran, and with the National Remote Sensing Laboratories, Iranian Space Agency. His research interests include assessment of biophysical and biochemical properties of forest canopies by fusing different remotely sensed data, particularly imaging spectroscopy and airborne laser scanning.

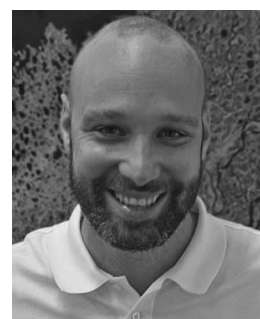

Felix Morsdorf received the M.Sc. degree in physical oceanography from the University of Kiel, Kiel, Germany, in 2001 and the Ph.D. degree in geography, with a specialization in LIDAR remote sensing, from the University of Zürich, Zürich, Switzerland, in 2007.

$\mathrm{He}$ is a group leader with the Department of Geography, University of Zürich. His group uses empirical and physical approaches to bridge the semantic gap between data and information. His research is focused on laser scanning and vegetation structure.

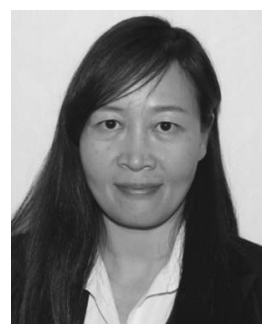

Lingli Zhu received the Master's and Ph.D. degrees in photogrammetry and remote sensing from Aalto University, Espoo, Finland, in 2007 and 2015, respectively.

She is currently a Research Manager and group leader with the Department of Remote Sensing and Photogrammetry, Finnish Geospatial Research Institute, Masala, Finland. She is a member of the Centre of Excellence in Laser Scanning Research granted by the Academy of Finland for the period of 2014-2019. Her research interests include 3-D modeling from laser scanning, virtual reality, photogrammetry, and remote sensing.

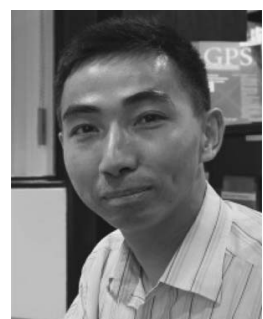

Jingbin Liu received the Ph.D. degree in geodesy and geomatics from Wuhan University, Wuhan, China, in 2008 .

$\mathrm{He}$ is a Specialist Research Scientist with the Department of Remote Sensing and Photogrammetry, Finnish Geospatial Research Institute, Masala, Finland. His research interests include indoor/ outdoor georeferencing, laser scanning point cloud processing, and innovative geospatial applications.

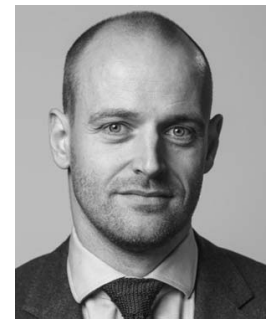

Petteri Alho received the Ph.D. degree in physical geography in 2005 from the University of Turku, Turku, Finland.

Since 2000, he has been with the Department of Geography and Geology, University of Turku, where he holds a professorship in hydrogeography and remote sensing. His main research interest is utilizing remotely sensed data to study the physical environment, particularly rivers and forests. 